\title{
ON THE ARITHMETIC OF PROJECTIVE COORDINATE SYSTEMS
}

\author{
BY \\ CHRISTIAN HERRMANN ${ }^{1}$
}

\begin{abstract}
A complete list of subdirectly irreducible modular (Arguesian) lattices generated by a frame of order $n \geq 4(n \geq 3)$ is given. Also, it is shown that a modular lattice variety containing the rational projective geometries cannot be both finitely based and generated by its finite dimensional members.
\end{abstract}

1. Introduction. Frames were introduced by von Neumann $[\mathbf{3 1}]$ as the abstract lattice theoretic counterpart of coordinate systems in projective geometry. His construction of a coordinate ring $R=R_{\phi}$ for a complemented modular lattice $L$ with a frame $\phi$ of order $n \geq 4$ extends to modular lattices in general (Artmann [2], Freese [11]). For $n \geq 3$ and lattices which are Arguesian in the sense of Jónsson [26] the ring construction is a recent achievement of Day and Pickering [8]. Proceeding towards a coordinatization of $L$ one finds for any subring $S$ of $R$ join homomorphisms of the submodule lattice $L\left({ }_{S} S^{2}\right)$ into $L$ which come from the canonical embeddings of $S^{2}$ into $R^{n}$ if $L=L\left({ }_{R} R^{n}\right)$. For Arguesian lattices this is contained in $[8]$, in essence.

For a completely primary and uniserial $S$ these maps extend to a join homomorphism of $L\left({ }_{S} S^{n}\right)$ into $L$. The proof uses the representation of automorphisms of ${ }_{S} S^{n}$ in $\operatorname{Aut}(L)$ for free $L$-an idea developed by Huhn [23] and Freese [11]. With the dual meet homomorphism and the method of "bounded homomorphisms" (McKenzie [30] and Wille [32]) one has a new approach to the coordinatization theorems for primary lattices-cf. Jónsson and Monk [26].

On the other hand with $S=Z_{p^{k}}$, the residue class ring of integers modulo $p^{k}$, one derives that the subgroup lattice $L\left(Z_{p^{k}}^{n}\right)$ is the only modular (Arguesian if $n=3$ ) subdirectly irreducible lattice generated by a frame of characteristic $p^{k}, p$ prime. The case $k=1$ has been solved by Freese [11] for $n \geq 4$ and by Day [7] for $n=3$. This is a basis for our main result. Let $Z_{p^{\infty}}$ denote the quasicyclic (Prüfer) $p$-group and $Q_{p}$ the rationals with denominator relatively prime to $p$.

THEOREM 1.1. The following is a complete list of subdirectly irreducible modular (Arguesian) lattices generated by a frame of order $n \geq 4(n \geq 3)$ :

(i) the $(n-1)$-dimensional rational projective geometry $L\left({ }_{Q} Q^{n}\right)$,

(ii) the subgroup lattices $L\left(Z_{p^{k}}^{n}\right)$, p prime, $k<\infty$,

(iii) the lattices $L_{c}\left(Z_{p^{\infty}}^{n}\right)$ of closed subgroups of $Z_{p^{\infty}}^{n}, p$ prime,

(iv) the duals of (iii), the lattices $L\left({ }_{Q_{p}} Q_{p}^{n}\right)$.

Received by the editors February 13, 1982 and, in revised form, August 10, 1983.

1980 Mathematics Subject Classification. Primary 06C05.

Key words and phrases. Modular lattices, frames.

${ }^{1}$ Supported by NRSEC Grant A-8190. 
This has been shown in Herrmann and Huhn [20] for lattices in the variety generated by all lattices of normal subgroups of groups. The generating frame can be chosen canonically in each instance. An immediate consequence is the following

COROLLARY 1.2. The word problem for finitely presented modular (Arguesian) lattices generated by a frame of order $n \geq 4(n \geq 3)$ is solvable.

In particular, a lattice relation involving the frame and integers of the coordinate ring, only, is valid in general if and only if it is valid in all $L\left(Z_{p^{k}}^{n}\right)$. That frame generated lattices play an important role in all word and classification problems for finitely generated modular lattices is shown in the following example which follows from the main result in Herrmann-Kindermann-Wille [21].

COROLlaRY 1.3. $D_{2}, M_{3}$, and the lattices from Theorem 1.1 with $n=3$ are exactly the subdirectly irreducible Arguesian lattices generated by elements $a, b \leq c$, $d \leq e$.

Also, each of the lattices in Theorem 1.1 is generated by four elements [17]. Unfortunately, there are subdirectly irreducible subgroup lattices of abelian groups which are generated by four elements but not by a frame-one of the reasons that the word problem for the free modular lattices on four generators is unsolvable [18]. As far as Arguesian lattices are concerned the key for a solution might be a suitable concept of "skew frames". Since notational problems would duplicate, at least, we avoid a discussion of such in this paper.

Frames and coordinate rings are also inherent in unsolvability results on modular lattices, only to mention Hutchinson's [24] and Freese's [12] results on word problems in five generators and Freese's result that the variety of modular lattices is not generated by its finite members $[\mathbf{1 0}]$. The latter can be strengthened a little bit. Let $M, M_{f}, M_{f d}$, and $M_{0}$ denote the lattice varieties generated by all, all finite, and all finite dimensional modular lattices and all subspace lattices of vector spaces over $Q$, respectively.

THEOREM 1.4. A modular lattice variety containing $M_{0}$ cannot be both finitely based and generated by its finite dimensional members.

COROLlaRY 1.5. Neither $M_{f}$ nor $M_{f d}$ are finitely based and $M_{f} \varsubsetneqq M_{f d} \varsubsetneqq M$.

COROLLARY 1.6. The variety of Arguesian lattices is not generated by its finite dimensional members.

The last corollary answers a question raised by Bjarni Jónsson. For the first observe that $M_{0} \subseteq M_{f}$ by Herrmann and Huhn [19] and that $M_{f} \varsubsetneqq M_{f d}$ by Freese [10].

As basic references we use Birkhoff [3], Crawley-Dilworth [4], Maeda [28], and von Neumann [31], for abelian group theory Fuchs [15]. A good introduction to frames and coordinatization is provided by forthcoming lecture notes of Alan Day [5].

I am highly indebted to Alan Day for contributing in many ways to this paper. Also I have to thank András Huhn and Ralph Freese whose seminar notes I used in rewriting the proof of Theorem 1.4 . 
2. Coordinatization by primary rings. Von Neumann [31] introduced a concept of coordinate system for a modular lattice with 0 and 1: A system $\phi=$ $\left(a_{i}, c_{i j} ; 1 \leq i \neq j \leq n\right)$ of elements of $L$ is a (normalized) $n$-frame of $L$ if $\sum a_{i}=1$, $a_{j} \sum_{i \neq j} a_{i}=0, c_{i j}=c_{j i}, a_{i} c_{i j}=0, a_{i}+c_{i j}=a_{i}+a_{j}$, and $c_{i k}=\left(a_{i}+a_{k}\right)\left(c_{i j}+c_{j k}\right)$. We write $x+y$ for the join and $x y$ for the meet of $x$ and $y$. The relations imply for $1 \neq 0$ that the $a_{i}$ are the atoms of a Boolean sublattice $2^{n}$ and that $0, a_{i}, c_{i j}, a_{j}$, and $a_{i}+a_{j}$ form a 5-element nondistributive sublattice $M_{3}$.

Given an (associative) ring with 1 and a free (unital left) $R$-module ${ }_{R} M$ with basis $e_{i}(1 \leq i \leq n)$ we get an $n$-frame of the lattice $L\left({ }_{R} M\right)$ of $R$-submodules by $a_{i}=R e_{i}, c_{i j}=R\left(e_{i}-e_{j}\right)$. Every $n$-frame of $L\left({ }_{R} M\right)$ arises in this way, all are related via automorphisms of ${ }_{R} M$. If the $e_{i}$ are the canonical basis vectors of $M=R^{n}$ we speak of the canonical $n$-frame.

Clearly, any permutation of indices yields an $n$-frame again.

LEMMA 2.1. Let $\Gamma$ describe a connected graph on $\{1, \ldots, n\}$ and let the elements $a_{i}(1 \leq i \leq n)$ and $c_{i j}$ of $L$ with $(i, j) \in \Gamma$ satisfy the relations of an $n$-frame as far as they make sense. Then this system can be extended to an $n$-frame of $L$.

PROOF. This is shown by iterated application of the following observation. Let $\phi$ be an $n$-frame of the interval $[0, u]$ and $a, c$ such that $u(a+c)=a_{n}$ and $a_{n}, c, a$ is a 2 -frame of $[0, a+c]$. Let $a_{n+1}=a, c_{n n+1}=c, c_{i n+1}=\left(a_{i}+a\right)\left(c_{n i}+c\right)$ for $i<n$ and $c_{n+1} i=c_{i n+1}$ for $i \leq n$. Then $\left(a_{i}, c_{i j} ; 1 \leq i, j \leq n+1\right)$ is an $n+1$-frame of $[0, u+a]$.

Indeed, we have $a u=a a_{n}=0$ whence $a_{1}, \ldots, a_{n+1}$ are independent [31, p. 9]. The remaining relations follow with $[\mathbf{3 1}$, p. 118].

An equivalent concept is Huhn's [22] diamonds. Following Day and Pickering [8] we call $\left(d_{1}, \ldots, d_{n+1}\right)$ a spanning $n$-diamond of $L$ if $\sum_{i \neq j} d_{i}=1$ and $d_{j} \sum_{i \neq j, k} d_{i}=0$ for all $j \neq k$. If $\phi$ is a 3 -frame and $i, j, k$ all distinct then $\left(c_{k j}, a_{k}, a_{i}, c_{i j}\right)$ is a spanning 3-diamond.

As one knows from projective geometry, for a coordinatization one needs $n \geq 4$ or Desargues' law. We use Jónsson's [26] lattice theoretic version. A triple $\mathbf{x}=$ $\left(x_{0}, x_{1}, x_{2}\right)$ of elements of a lattice is called a triangle and normal if

$$
x_{2}=\left(x_{0}+x_{2}\right)\left(x_{1}+x_{2}\right) \text {. }
$$

Two triangles $\mathbf{x}$ and $\mathbf{y}$ are centrally perspective $(\mathrm{CP})$ if

$$
\left(x_{0}+y_{0}\right)\left(x_{1}+y_{1}\right) \leq x_{2}+y_{2}
$$

and axially perspective (AP) if

$$
\left(x_{0}+x_{1}\right)\left(y_{0}+y_{1}\right) \leq\left(x_{0}+x_{2}\right)\left(y_{0}+y_{2}\right)+\left(x_{1}+x_{2}\right)\left(y_{1}+y_{2}\right) .
$$

A modular lattice is called Arguesian if every CP pair of triangles is AP. This implication can be stated as a lattice identity determining a self-dual lattice variety (Jónsson [27]). In an Arguesian lattice one has that AP implies CP for pairs of normal triangles. Every lattice of submodules is Arguesian. This extends to congruence lattices of algebraic structures in a congruence modular variety, Freese and Jónsson $[\mathbf{1 3}]$. 
A general assumption for this paper is that $\phi$ is an $n$-frame of the modular lattice $L$ and either $n \geq 4$, or $n \geq 3$ and $L$ Arguesian. For $i, j, k$ all distinct let

$$
\begin{aligned}
& R_{i j}=R_{i j \phi}=\left\{x \in L \mid a_{j} x=0, a_{j}+x=a_{i}+a_{j}\right\}, \\
& \pi_{i j k} x=\left(x+c_{j k}\right)\left(a_{i}+a_{k}\right), \\
& x \oplus_{i j k} y=\left(a_{i}+a_{j}\right)\left[\left(x+a_{k}\right)\left(c_{i k}+a_{j}\right)+\pi_{j i k} y\right], \\
& x \ominus_{i j k} y=\left(a_{i}+a_{j}\right)\left[a_{k}+\left(c_{j k}+x\right)\left(a_{j}+\pi_{i j k} y\right)\right], \\
& x \otimes_{i j k} y=\left(a_{i}+a_{j}\right)\left(\pi_{i j k} x+\pi_{j i k} y\right) .
\end{aligned}
$$

THEOREM 2.2. The $R_{i j}$ are associative rings with zero $a_{i}$, unit $c_{i j}$, addition $\oplus_{i j k}$, difference $\ominus_{i j k}$, and multiplication $\otimes_{i j k}$. These operations do not depend on k. Moreover, $R_{i j}$ is isomorphic to $R_{i k}$ and $R_{k j}$ via $\pi_{i j k}$ and $\pi_{j i k}$, respectively,.

This is von Neumann [31] for $n \geq 4$ and Day-Pickering [8] for $n=3$. Just note that von Neumann uses the opposite multiplication.

Also, observe that for $L=L\left({ }_{R} R^{n}\right)$ the map $r \mapsto R\left(e_{i}-r e_{j}\right)$ is an isomorphism of $R$ onto $R_{i j}$. The projective isomorphisms $\pi_{i j k} \uparrow R_{i j}$ allow us to speak of the coordinate ring $R_{\phi} \cong R_{i j}$ with $0,1, \oplus, \ominus, \otimes$ and write $r=r_{i j} \in R_{i j}$ for $r$ in $R_{\phi}$. Then the multiplication formula reads $(r \otimes s)_{i j}=\left(a_{i}+a_{j}\right)\left(r_{i k}+s_{k j}\right)$. Where no confusion with lattice operations is possible we write $r+s, r-s$ and $r s$. Recall from Freese [10, Lemma 2.3], that $r$ is invertible in $R_{\phi}$ if and only if $r_{i j} \in R_{j i}$ and then

$$
\begin{array}{cc}
1 / r_{i j}=r_{j i}, & \ominus 1_{i j}=\ominus 1_{j i} \\
r_{i k}+s_{k j}=r_{i k}+r \otimes s_{i j}, & s_{i k}+r_{k j}=s \otimes r_{i j}+r_{k j} .
\end{array}
$$

DEFINITION. For a sequence $\mathbf{r}=\left(r^{1}, \ldots, r^{n}\right)$ in $R_{\phi}^{n}$ let

$$
a_{i}^{*}=\sum_{j \neq i} a_{j}, \quad \mathbf{r}_{i}=\mathbf{r}_{i \phi}=\prod_{j \neq i}\left(a_{i}^{*} a_{j}^{*}+r_{i j}^{j}\right), \quad \mathbf{r}_{\phi}=\prod\left(a_{i}+\mathbf{r}_{i}\right) .
$$

In the model $L\left({ }_{R} R^{n}\right)$ one has $\mathbf{r}_{i}=R\left(\sum_{j \neq i} r^{j} e_{j}-e_{i}\right)$ and $\mathbf{r} \geq R \sum r^{i} e_{i}$. Equality holds for uniserial $R$. Namely, let, e.g., $r^{i} \in r^{1} R$ for all $i, r^{i}=r^{1} s^{i}$. Then $\left(\mathbf{r}_{2}+a_{2}\right)\left(\mathbf{r}_{3}+a_{3}\right)$ consists of all vectors $\mathbf{t}$ with $t^{i}=x r^{i}=x r^{1} s^{i}$ for $i \neq 2$ and $t^{i}=y r^{i}=y r^{1} s^{i}$ for $i \neq 3, x, y$ in $R$. Since $x r^{1}=y r^{1}$ these are exactly the elements of $R \sum r^{i} e_{i}$.

PROPOSITION 2.3.

$$
a_{i}+a_{k}+\mathbf{r}_{i}=a_{i}+\prod_{j \neq i, k}\left(a_{i}^{*} a_{j}^{*}+r_{i j}^{j}\right)=a_{i}+a_{k}+\mathbf{r}_{k}
$$

and $a_{i}+a_{k}+r_{i j}=a_{i}+a_{k}+r_{k j}$. If $r^{k}=0$ then $\mathbf{r}_{\phi}=a_{k}^{*}\left(a_{k}+\mathbf{r}_{k}\right)$. have

PROOF. By the projective isomorphisms of $\left[0, a_{k}^{*}\right]$ onto $\left[a_{k}, 1\right]$ and $\left[c_{k i}, 1\right]$ we

$$
\begin{aligned}
a_{i}+a_{k}+\mathbf{r}_{i} & =a_{i}+\left(a_{i}^{*} a_{k}^{*}+r_{i k}^{k}+a_{k}\right) \prod_{j \neq i, k}\left(a_{i}^{*} a_{j}^{*}+r_{i j}^{j}\right) \\
& =a_{i}+\prod_{j \neq i, k}\left(a_{i}^{*} a_{j}^{*}+r_{i j}^{j}\right)=a_{i}+a_{k}+\prod_{j \neq i, k}\left(a_{k}^{*} a_{i}^{*} a_{j}^{*}+r_{i j}^{j}\right) \\
& =a_{i}+a_{k}+\prod_{j \neq i, k}\left(a_{k}^{*} a_{i}^{*} a_{j}^{*}+c_{k i}+r_{i j}^{j}\right) .
\end{aligned}
$$


Due to (2.2) we may replace $c_{k i}+r_{i j}^{j}$ by $c_{k i}+r_{k j}^{j}$ yielding the last expression as $a_{i}+a_{k}+\mathbf{r}_{k}$ by symmetry. Now, if $r^{k}=0$ then

$$
\begin{aligned}
a_{i}+\mathbf{r}_{i} & =a_{i}+\left(a_{i}^{*} a_{k}^{*}+a_{i}\right) \prod_{j \neq i, k}\left(a_{i}^{*} a_{j}^{*}+r_{i j}^{j}\right) \\
& =a_{k}^{*}\left(a_{i}+a_{k}+\mathbf{r}_{i}\right)=a_{k}^{*}\left(a_{i}+a_{k}+\mathbf{r}_{k}\right) .
\end{aligned}
$$

In the sequel let $S$ be a unitary subring of $R$ and $S^{(i, j)}$ the submodule of ${ }_{S} S^{n}$ consisting of all $\mathbf{s}$ with $s^{k}=0$ for $k \neq i, j$.

LEMMA 2.4. There is a join homomorphism $\sigma$ of the semilattice of finitely generated $S$-submodules of $S^{(i, j)}$ into the interval $\left[0, a_{i}+a_{j}\right]$ of $L$ such that $\sigma S \mathbf{r}=$ $\mathbf{r}_{\phi}$ for all $\mathbf{r}$ in $S^{(i, j)}$.

For Arguesian lattices the adjoint meet homomorphism has been considered in $\S 5$ of Day-Pickering [8]-even for hyperplanes $\left[0, a_{i}^{*}\right]$. For $n \geq 4$ the coordinatization still works for "codimension 2 " intervals $\left[0, a_{i}^{*} a_{j}^{*}\right]$. As a substitute for the Arguesian law we introduce the maps

$$
\alpha_{i j h}^{r} x=\left[\left(x+a_{h}\right)\left(a_{i}^{*} a_{h}^{*}+c_{i h}\right)+r_{h j}\right] a_{h}^{*}, \quad \mu_{r i h} x=\left[\left(x+r_{h i}\right) a_{i}^{*}+c_{i h}\right] a_{h}^{*}
$$

for distinct $i, j, k$ and $r$ in $R_{\phi}-\mathrm{cf}$. Artmann [2].

LEMMA 2.5. $\alpha_{i j h}^{r}$ is an automorphism of $\left[0, a_{h}^{*}\right]$ mapping $s_{i j}$ onto $s \oplus r_{i j}$ and fixing all $x$ with $x \geq a_{j}$ or $x \leq a_{i}^{*}$. $\mu_{\text {rih }}$ is a meet endomorphism of $\left[0, a_{h}^{*}\right]$ with $\mu_{r i h} s_{i k}=r \otimes s_{i k}$ for $k \neq i, h, \mu_{r i h} x \geq x$ for $x \leq a_{i}^{*}$ and fixpoints $x \geq a_{i}$. If $r$ is invertible $\mu_{\text {rih }}$ is an automorphism and $\mu_{r i h} s_{k i}=(s / r)_{k i}$.

For the proof consider these maps as products of lattice translation maps. Still, we have to prove Lemma 2.4. First

$$
\begin{aligned}
\left(a_{j}+a_{k}\right)\left[\left(a_{k}+s_{i j}\right)\left(a_{j}+t_{i k}\right)+\left(a_{k}+u_{i j}\right)\left(a_{j}+v_{i k}\right)\right] & \\
= & \left(a_{j}+a_{k}\right)\left[a_{i}+\left(a_{k}+s \ominus u_{i j}\right)\left(a_{j}+t \ominus v_{i k}\right)\right]
\end{aligned}
$$

for $i, j, k$ distinct. This is Lemma 5.1 of $[8]$ for $n=3$. For $n \geq 4$ let $h \neq i, j, k$ and apply $\alpha_{i k h}^{-v} \alpha_{i j h}^{-u}$ to the left-hand side. It is both a fixpoint and mapped onto the right-hand side. Also,

$$
a_{i}+\left(a_{k}+s_{i j}\right)\left(a_{j}+t_{i k}\right) \geq a_{i}+\left(a_{k}+r \otimes s_{i j}\right)\left(a_{j}+r \otimes t_{i k}\right),
$$

which is Theorem 5.3 of $[8]$ for $n=3$ and follows by application of $\mu_{\text {rih }}$ to the left-hand side for $n \geq 4$. Recall that for $\mathbf{s}$ in $S^{(i, j)}$ one has

$$
\mathbf{s}_{\phi}=\left(a_{i}+a_{j}\right)\left[a_{k}+\left(a_{j}+s_{k i}^{i}\right)\left(a_{i}+s_{k j}^{j}\right)\right]
$$

by Proposition 2.3. Hence $(r \mathbf{s})_{\phi} \leq \mathbf{s}_{\phi}$ by (2.4) and $(\mathbf{r} \ominus \mathbf{s})_{\phi} \leq \mathbf{r}_{\phi}+\mathbf{s}_{\phi}$ by (2.3). Consequently $\sigma U=\sum\left(\mathbf{r}_{\phi} \mid \mathbf{r} \in E\right), E$ a generating set of the $S$-module $U$, defines a join preserving map.

From (2.4) and (2.3) we derive

$$
\begin{aligned}
\left(a_{j}+a_{k}\right)\left[t_{i k}+\left(a_{k}+r_{i j}\right)\right. & \left.\left(a_{j}+t \ominus r \otimes s_{i k}\right)\right] \\
= & \left(a_{j}+a_{k}\right)\left[a_{i}+\left(a_{k}+r_{i j}\right)\left(a_{j}+\ominus r \otimes s_{i k}\right)\right] \\
\leq & \left(a_{j}+a_{k}\right)\left[a_{i}+\left(a_{k}+c_{i j}\right)\left(a_{j}+\ominus s_{i k}\right)\right] \\
& =\left(a_{j}+a_{k}\right)\left(c_{i j}+s_{i k}\right)=s_{j k},
\end{aligned}
$$


whence

$$
\left(a_{k}+r_{i j}\right)\left(t_{i k}+s_{j k}\right) \geq\left(a_{k}+r_{i j}\right)\left(a_{j}+t \ominus r \otimes s_{i k}\right),
$$

and, since both sides are complements of $a_{k}$ in $\left[0, a_{k}+r_{i j}\right]$,

$$
\left(a_{k}+r_{i j}\right)\left(t_{i k}+s_{j k}\right)=\left(a_{k}+r_{i j}\right)\left(a_{j}+t \ominus r \otimes s_{i k}\right),
$$

which is Lemma 10.6 in [31, p. 172] for $t=0$.

LEMMA 2.6. There is an $n$-frame $\phi^{*}$ of the dual lattice $L^{*}$ such that $a_{i}^{*}=$ $\sum_{j \neq i} a_{j}$ and $c_{1 j}^{*}=c_{1 j}+a_{1}^{*} a_{j}^{*}$. Moreover, $r \mapsto r_{j 1}^{*}=r_{1 j}+a_{1}^{*} a_{j}^{*}$ describes an isomorphism of the opposite of $R_{\phi}$ onto the coordinate ring $R_{\phi *}$ of $L^{*}$.

ProOF. That the $a_{i}^{*}$ and $c_{i j}^{*}$ give rise to an $n$-frame of $L^{*}$ is clear by Lemma 2.1. Let, e.g., $j=2$. By modularity, $r_{12} \mapsto r_{21}^{*}$ is a bijection of $R_{12}$ onto $R_{21}^{*}$ matching the zeros. We express the operations on $R_{21}^{*}$ in terms of $L$. Using the isomorphism between $\left[0, a_{1}+a_{2}+a_{3}\right]$ and $\left[a_{1}^{*} a_{2}^{*} a_{3}^{*}, 1\right]$ we have

$$
\begin{aligned}
r^{*} \ominus^{*} s_{21}^{*}= & a_{1}^{*} a_{2}^{*}+a_{3}^{*}\left[c_{13}^{*} r_{21}^{*}+a_{1}^{*}\left(s_{21}^{*} c_{13}^{*}+a_{2}^{*} a_{3}^{*}\right)\right]=a_{1}^{*} a_{2}^{*}+\left(a_{1}+a_{2}\right) \\
& \times\left[\left(c_{13}+a_{2}\right)\left(r_{12}+a_{3}\right)+\left(a_{2}+a_{3}\right)\left(\left(s_{12}+a_{3}\right)\left(c_{13}+a_{2}\right)+a_{1}\right)\right] \\
= & a_{1}^{*} a_{2}^{*}+r \oplus(\ominus s)_{12}=(r \ominus s)_{21}^{*},
\end{aligned}
$$

since by $(2.3)$,

$$
\begin{aligned}
\left(a_{2}+a_{3}\right)\left[\left(s_{12}+\right.\right. & \left.\left.a_{3}\right)\left(c_{13}+a_{2}\right)+a_{1}\right]=\left(a_{2}+a_{3}\right)\left(c_{13}+\ominus s_{12}\right)=\ominus s_{32} . \\
r^{*} \otimes^{*} s_{21}^{*}= & a_{1}^{*} a_{2}^{*}+\left(r_{21}^{*} c_{13}^{*}+a_{2}^{*} a_{3}^{*}\right)\left(s_{21}^{*} c_{23}^{*}+a_{1}^{*} a_{3}^{*}\right) \\
= & a_{1}^{*} a_{2}^{*}+\left[\left(r_{12}+a_{3}\right)\left(c_{31}+a_{2}\right)+a_{1}\right] \\
& \times\left[\left(s_{12}+a_{3}\right)\left(a_{1}+\left(c_{12}+a_{3}\right)\left(c_{13}+a_{2}\right)\right)+a_{2}\right] \\
= & a_{1}^{*} a_{2}^{*}+\left(\ominus r_{32}+a_{1}\right)\left[\left(s_{12}+a_{3}\right)\left(a_{1}+\ominus 1_{23}\right)+a_{2}\right] \\
= & a_{1}^{*} a_{2}^{*}+\left(\ominus r_{32}+a_{1}\right)\left(s_{13}+a_{2}\right) \\
= & a_{1}^{*} a_{2}^{*}+\left(a_{3}+s \otimes r_{12}\right)\left(s_{13}+a_{2}\right) \\
= & a_{1}^{*} a_{2}^{*}+s \otimes r_{12}=(s \otimes r)_{21}^{*}
\end{aligned}
$$

follows using (2.5) four times.

A key idea in Huhn [23] and Freese [11] was the representation of a linear group over a prime field in the automorphism group of a modular lattice freely generated by a frame (of prime characteristic). This works in greater generality. The basis is the elementary automorphisms of ${ }_{S} S^{n}$ given by $\alpha_{i j}^{r} e_{i}=e_{i}-r e_{j}, \mu_{q i} e_{i}=q^{-1} e_{i}$, $\alpha_{i j}^{r}\left(e_{k}\right)=\mu_{q i}\left(e_{k}\right)=e_{k}$ for $k \neq i$, where $r$ and $q$ are in $S$ and $q$ is invertible. The rings $S$ to be considered are completely primary and uniserial: there is a two-sided ideal $P$ such that every left or right ideal of $S$ is a power of $P$. In particular, $S r=r S$ for each $r$ and $P^{n}=0$ for some $m$. Of course, $L$ has to be free in some sense. Call $F$ the free resolution of $L$ over $\phi$ and $S, S$ a subring of $R_{\phi}$, if $F$ is the free lattice in the lattice variety generated by $L$ with generating set $\phi \cup\left\{r_{12} \mid r \in S\right\}$, the relations defining an $n$-frame, and all the relations $r \oplus s_{12}=t_{12}, r \otimes s_{12}=u_{12}$ where $r, s, t, u$ are in $S$ and $r+s=t, r s=u$ in $S$. By construction, $S$ is a subring of the coordinate ring of $\phi$ in $F$, too, and we have a canonical homomorphism of $F$ into $L$. If this is an isomorphism we say that $\phi$ is free in $L$ over $S$. 
THEOREM 2.7. Let $\phi$ be free in $L$ over a completely primary and uniserial $S \subseteq R_{\phi}$. Then there is a homomorphism $\psi \mapsto \psi_{\phi}$ of the automorphism group of ${ }_{S} S^{n}$ into that of $L$ such that $(\psi \mathbf{r})_{\phi}=\psi_{\phi}\left(\mathbf{r}_{\phi}\right)$ for all $\mathbf{r}$ in $S^{n}$.

PREVIEW OF PROOF. Since $S$ is local, every invertible $S$-matrix can be transformed into the identity matrix via elementary (Gaussian) transformations. Thus, every automorphism $\psi$ of ${ }_{S} S^{n}$ is a product of elementary ones-cf. [29, Theorem I.10]. In $\S 4$ we define for every elementary $\psi$ an automorphism $\psi_{\phi}$ of $L$ and show that $(\psi \mathbf{r})_{\phi}=\psi_{\phi}\left(\mathbf{r}_{\phi}\right)$ for all $\mathbf{r}$ in $S^{n}$. Thus, for arbitrary $\psi$ one can define $\psi_{\phi}=\prod \psi_{i \phi}$, choosing elementary $\psi_{i}$ such that $\psi=\prod \psi_{i}$. Since the $\mathbf{r}$ generate $L$ by hypothesis, $\psi_{\phi}$ does not depend on the choice of the $\psi_{i}$ and $\psi \mapsto \psi_{\phi}$ is a group homomorphism.

THEOREM 2.8. Let $S \subseteq R_{\phi}$ be completely primary and uniserial. Then there is a join homomorphism $\sigma$ of $L\left({ }_{S} S^{n}\right)$ into $L$ such that $\sigma S \mathbf{r}=\mathbf{r}_{\phi}$ for all $\mathbf{r}$ in $S^{n}$.

Proof. Of course, we may assume $\phi$ is free in $L$ over $S$. We show that for $\mathbf{r}, \mathbf{s}$ in $S^{n}$ there are $i, j$ and an automorphism $\psi$ such that $\psi \mathbf{r}$ and $\psi \mathbf{s}$ are in $S^{(i, j)}$. In view of Lemma 2.4 and Theorem 2.7, and the fact that every submodule of ${ }_{S} S^{n}$ is the join of finitely many cyclic ones, this suffices for proving that $\sigma U=\sum\left(\mathbf{r}_{\phi} \mid \mathbf{r} \in U\right)$ defines a join homomorphism of $L\left({ }_{S} S^{n}\right)$ into $L$. Indeed, since $S$ is right uniserial there is an $i$ with $r^{k}=r^{i} t^{k}$ for all $k$. Then with $\chi=\prod_{k \neq i} \alpha_{i k}^{t^{k}}$, the vector $\mathbf{r}$ has all but the $i$ th component zero. Similarly, we get $j$ and $\varphi$, fixing $\chi \mathbf{r}$ such that $\varphi \chi \mathbf{s}$ has $k$ th component zero for $k \neq i, j . \psi=\varphi \chi$ is the desired automorphism.

THEOREM 2.9. Let $L$ be subdirectly irreducible and generated by $\phi$ and the $s_{12}$ $(s \in S), S \subseteq R_{\phi}$, completely primary and uniserial. Then $L$ and $L\left({ }_{S} S^{n}\right)$ are isomorphic.

Proof. $S$ and its opposite $T$ are local, artinian, and uniserial. Let $\sigma$ be given according to Theorem 2.8. By 2.6 and the dual of 2.8 there is a join homomorphism $\tau$ of $L\left({ }_{T} T^{n}\right)$ into $L^{*}$ mapping $T\left(e_{1}-r e_{j}\right)$ onto $r_{j 1 \phi^{*}}$. Now, $S$ has the double annihilator property and the bimodule ${ }_{S} S_{S}$ defines a Morita duality between finitely generated left and right $S$-modules--see [1, Exercise 24.10-13]. Identifying right $S$ modules with left $T$-modules one has a dual isomorphism $\delta$ of $L\left({ }_{S} S^{n}\right)$ onto $L\left({ }_{T} T^{n}\right)$. Due to the transitive action of the automorphism group on the set of $n$-frames, we may assume $\delta$ maps the dual $n$-frame $\phi^{\prime *}$ associated with the canonical frame $\phi^{\prime}$ of $L\left({ }_{S} S^{n}\right)$ onto the canonical $n$-frame of $L\left({ }_{T} T^{n}\right)$, and $r_{j 1 \phi^{\prime *}}$ onto $T\left(e_{1}-r e_{j}\right)$. Then $\gamma=\delta \tau$ is a meet homomorphism of $L\left({ }_{S} S^{n}\right)$ into $L$ mapping $\phi^{\prime *}$ onto $\phi^{*}$ and $r_{j 1 \phi^{\prime *}}$ onto $r_{j 1 \phi^{*}}$. Hence

$$
\gamma a_{i}^{\prime}=\gamma \prod_{j \neq i} a_{j}^{\prime *}=a_{i} \quad \text { and } \quad \gamma r_{1 j \phi^{\prime}}=\gamma\left(\prod_{i \neq 1, j} a_{i}^{\prime *} r_{j 1 \phi^{\prime *}}\right)=r_{1 j \phi}
$$

Thus, one has $\sigma x \leq \gamma x$ on the generating set $\phi \cup\left\{r_{1 j} \mid r \in S\right\}$ of $L\left({ }_{S} S^{n}\right)$, whence for all $x$. Since $L$ and $L\left({ }_{s} S^{n}\right)$ are subdirectly irreducible (see Theorems 6.7 and 6.2 in [26]) and since $L$ is generated by the image of $\sigma$, either $\sigma=\gamma$ is an isomorphism or $\sigma p \leq \gamma q$ for every prime quotient $p / q$ in $L\left({ }_{s} S^{n}\right)-$ cf. Proposition 1 of the Appendix. The latter is impossible. Namely, choose $k$ minimal with $P^{k}=0$ and $r$ 
with $S r=P^{k-1}$. Then $S e_{1}+P^{k-1} e_{2}$ covers $S e_{1}$ in $L\left({ }_{S} S^{n}\right)$ but $\sigma\left(S e_{1}+P^{k-1} e_{2}\right)=$ $a_{1}+r_{12}>a_{1}=\gamma S e_{1}$ since $r_{12} \leq a_{1}$ would imply $r_{12}=a_{1}$ and $r=0$.

We say that $\phi$ has characteristic $m$ if the additive order of 1 in $R_{\phi}$ is $m$. With $S=Z_{p^{k}}$ we have

COROLLARY 2.10. Let $L$ be subdirectly irreducible and generated by $\phi$ of characteristic $p^{k}$, p prime. Then $L$ is isomorphic to $L\left(Z_{p^{k}}^{n}\right)$.

Also, one easily derives the coordinatization theorem of Jónsson and Monk [26] for primary lattices in the special case that the unit is a join of independent cycles of equal length.

3. Reduction of frames. In this section we introduce some basic manipulations with frames. Again, let $L$ be a modular lattice with $n$-frame $\phi$ and coordinate ring $R_{\phi}$. For any integer $r$, we have a corresponding element in $R_{\phi}$ and we may use the notation $r_{i j} \in R_{i j \phi}$.

LEMмA 3.1. Let $k$ be fixed and $u_{k} \leq a_{k}$. Define $u_{i}=a_{i}\left(u_{k}+c_{k i}\right)$ for $i \neq k$ and $u=\sum u_{i}$. Then

$$
\phi_{u}=\left(u a_{i}, u c_{i j} ; 1 \leq i \neq j \leq n\right) \quad \text { and } \quad \phi^{u}=\left(u+a_{i}, u+c_{i j} ; 1 \leq i \neq j \leq n\right)
$$

are $n$-frames of the interval sublattices $[0, u]$ and $[u, 1]$ of $L$. For every integer $r$ one has

$$
u r_{i j}=r_{i j \phi_{u}}, \quad u+r_{i j}=r_{i j \phi} u,
$$

This stems from $\S 1$ in Freese [10]. $\quad \square$ We say that $\phi_{u}$ arises from $\phi$ by reduction with $u_{k}$. This construction is compatible with the projective isomorphisms $\pi_{i j}$ as the following shows.

LEMMA 3.2. $u$ and the $a_{i}$ generate a distributive sublattice. If $u_{k} \leq v_{k} \leq a_{k}$ then $u \leq v,\left(\phi_{v}\right)_{u}=\phi_{u},\left(\phi^{u}\right)^{v}=\phi^{v}$, and $\left(\phi^{u}\right)_{v}=\left(\phi_{v}\right)^{u}$. Moreover,

$$
\begin{aligned}
& u a_{i}=u_{i}=a_{i}\left(u+q_{i j}\right)=a_{i}\left(u_{j}+q_{i j}\right) \quad \text { for } q= \pm \mathbf{1}, \\
& r_{i j}\left(u+a_{j}\right)=u r_{i j} \text { for integer } r, \\
& \left(x+c_{j h}\right)\left(a_{i}+a_{h}\right)=\left(x+u c_{j h}\right)\left(u a_{i}+u a_{h}\right) \quad \text { for } x \leq u\left(a_{i}+a_{j}\right) .
\end{aligned}
$$

PROOF. $u \sum\left(a_{i} \mid i \in I\right)=\sum\left(u_{i} \mid i \in I\right)$ easily follows from the independence of the $a_{i}$. The next claim is obvious. By (2.1) and (2.2) one has for $i, j \neq k$

$$
\begin{gathered}
a_{i}\left(u_{j}+q_{i j}\right)=a_{i}\left(u_{k}+c_{j k}+q_{i j}\right)=a_{i}\left(u_{k}+c_{i k}+q_{j k}\right) \\
=a_{i}\left(u_{k}+c_{i k}+\left(a_{i}+a_{k}\right) q_{j k}\right)=u_{i}, \\
a_{i}\left(u_{k}+q_{i k}\right)=a_{i}\left(u_{k}+c_{i k}+q_{k j}\right)=u_{i},
\end{gathered}
$$

and

$$
a_{k}\left(u_{j}+q_{k j}\right)=a_{k}\left(a_{j}\left(u_{k}+q_{k j}\right)+q_{k j}\right)=a_{k}\left(u_{k}+q_{k j}\right)=u_{k} .
$$

Since $a_{i}\left(u+q_{i j}\right)=u_{i}+a_{i}\left(u_{j}+q_{i j}\right)$, this settles (3.2).

Now,

$$
q_{i j}\left(u+a_{j}\right)=q_{i j}\left(u_{i}+a_{j}\right) \leq u_{i}+a_{j}\left(u_{i}+q_{i j}\right) \leq u_{i}+u_{j}
$$

and, by induction,

$$
\begin{aligned}
r \oplus q_{i j}\left(u+a_{j}\right) & =\left(\left(r_{i j}+a_{h}\right)\left(c_{i h}+a_{j}\right)+q_{j h}\right)\left(u+a_{j}+a_{h}\right)\left(a_{i}+a_{j}\right) \\
& =\left(\left(u r_{i j}+a_{h}\right)\left(u c_{i h}+a_{j}\right)+q_{j h}\right)\left(u+a_{j}\right)\left(a_{i}+a_{j}\right)=r \oplus q_{i j \phi_{u}} .
\end{aligned}
$$


Finally,

$$
\begin{aligned}
\left(x+c_{j h}\right)\left(a_{i}+a_{h}\right) & =\left(x+c_{j h}\left(a_{i}+c_{j h}\left(a_{i}+u_{j}+a_{h}\right)\right)\right)\left(a_{i}+a_{h}\right) \\
& =\left(x+u c_{j h}\right)\left(u_{i}+u_{h}\right) .
\end{aligned}
$$

Two kinds of reductions are of particular interest. For $r$ in $R$ let $\tilde{r}$ and the reduced frame $\phi_{r}$ arise from $a_{1} r_{12}$ and $\bar{r}$ arise from $a_{1}\left(a_{2}+r_{21}\right)$. In the model $L\left({ }_{R} R^{n}\right)$ we have $\tilde{r}=\{\mathbf{s} \mid \mathbf{s} r=0\}$ and $\bar{r}=R^{n} r$.

LEMMA 3.3. Let $r, s$ be in $R$. Then

$$
\begin{aligned}
& r_{i j \phi_{r}}=a_{i \phi_{r}}=\tilde{r} a_{i}=a_{i} r_{i j}=\left(\tilde{r} a_{i}\right)_{\phi_{r s}} \quad \text { and } \quad \tilde{r} \leq \tilde{r s} \\
& s \otimes r_{i j} \leq a_{i}+\bar{r} a_{j}, \quad \overline{s r} \leq \bar{r}, \quad\left(\bar{r} a_{i}\right)_{\phi} \overline{s r}=\bar{r} a_{i}+\overline{s r} \\
& s_{i j}\left(\tilde{r}+a_{i}\right) \leq \tilde{s r} a_{i}+\tilde{r} a_{j}, \\
& \bar{r} a_{j}=\left(a_{i}+r_{i j}\right) a_{j}=\left(a_{i}+\ominus r_{i j}\right) a_{j}=\overline{-r} a_{j}, \\
& \tilde{r} \leq \bar{s} \quad \text { if } a_{i} \tilde{r}=a_{i} \bar{s} \text { for some } i, \\
& \mathbf{s}_{\phi} \leq \bar{r} \quad \text { if } s^{j} \in R r \text { for all } j .
\end{aligned}
$$

PROOF.

$$
a_{i} \tilde{r s}=a_{i}\left(r_{i k}+s_{k j}\right) \geq a_{i} r_{i k}=a_{i} \tilde{r}
$$

and

$$
s \otimes r_{i j} \leq\left(a_{i}+a_{j}\right)\left(a_{i}+a_{k}+r_{k j}\right)=a_{i}+r a_{j} .
$$

The remaining claims of (3.5) and (3.6) follow with (3.1) and (3.4). Next,

$$
s_{i j}\left(a_{i}+\tilde{r}\right) \leq\left(s_{i j}+r_{j k}\right)\left(a_{i}+\tilde{r} a_{j}\right)=\left(s_{i j}+r_{j k}\right) a_{i}+\tilde{r} a_{j}=\tilde{s r} a_{i}+\tilde{r} a_{j} .
$$

(3.8) is a consequence of Lemma 2.4 ; (3.9) follows from (3.4). (3.10) follows with (3.6), Lemma 3.2, and the independence of the $a_{i}$.

(3.1) implies

COROLLARY 3.4. If $r \neq 0$ is an integer than $\phi_{r}$ and $\phi^{\bar{r}}$ have characteristic dividing $r$.

LEMMA 3.5. Let $L$ be generated by $\phi$ and the $s_{12}(s \in S), S$ a subring of $R_{\phi}$. If $S$ has a proper central idempotent, then $L$ has a proper direct decomposition.

This is taken from Day [6]. We just give a proof for the case that $L$ is generated by $\phi$. Let $e$ be an idempotent, $u_{2}=a_{2} \bar{e}$, and $v_{2}=a_{2} \overline{1-e}$. Then $u_{2}+v_{2} \geq$ $a_{2}\left(c_{12}+a_{1}\right)=a_{2}$ by Lemma 2.4. Also, by definition of addition,

$$
\begin{aligned}
a_{1}+1 \ominus e_{12} & =\left(a_{1}+a_{2}\right)\left[\left(c_{12}+a_{3}\right)\left(c_{13}+a_{2}\right)+\ominus e_{32}+a_{1}\right] \\
& =\left(a_{1}+a_{2}\right)\left(c_{23}+a_{1}+\ominus e_{32}\right) .
\end{aligned}
$$

Since $e_{12} \leq e_{13}+e_{32}$ by idempotency, it follows that

$$
\begin{aligned}
u_{2} v_{2} & \leq a_{2}\left(a_{1}+e_{13}+e_{32}\right)\left(a_{1}+\ominus \mathbf{1}_{23}+e_{32}\right) \\
& =a_{2}\left(e_{32}+\ominus \mathbf{1}_{23}\left(a_{1}+e_{13}+e_{32}\right)\right) \\
& =a_{2}\left(e_{32}+\ominus \mathbf{1}_{23} e_{32}\right)=a_{2} e_{32}=0 ;
\end{aligned}
$$

cf. [14]. Hence $u$ and $v$ decompose the frame and are complementary central elements of $L-\mathrm{cf}$. $[\mathbf{2 0}, 2.2]$.

We conclude this section with more details about the expressions $\mathbf{s}_{\phi}$, where $\mathrm{s} \in S^{n}, S$ a subring of $R_{\phi}$. 
LEMMA 3.6. If $n \geq 4$ and the $a_{1} \tilde{s}$ ( $s$ in $S, s \neq 0$ ) generate a distributive sublattice of $L$, then

$$
\mathbf{s}_{\phi}=\left(a_{i}+a_{j}+\mathbf{s}_{j}\right) \prod_{k \neq i, j}\left(a_{k}+\mathbf{s}_{k}\right) .
$$

PROOF. In view of the projective isomorphisms $\pi_{1 i}$ we may assume $i=1$ without loss of generality. Observe that $a_{1} \tilde{0}=a_{1}$. Now, by symmetry and Proposition 2.3 it suffices to show $X=\prod_{1<k}\left(a_{1}+a_{k}+\mathbf{s}_{1}\right) \leq a_{1}+\mathbf{s}_{1}$. Since $a_{k}+\mathbf{s}_{1}=\prod_{1<j \neq k}\left(a_{1}^{*} a_{j}^{*}+s_{1 j}^{j}\right)$ we have

$$
\begin{aligned}
X \leq & a_{1}+\left(a_{2}+\mathbf{s}_{1}\right)\left(a_{1}+a_{3}+\mathbf{s}_{1}\right)\left(a_{1}+a_{4}+\mathbf{s}_{1}\right) \\
= & a_{1}+\prod_{2<j}\left(a_{1}^{*} a_{j}^{*}+s_{1 j}^{j}\right)\left[a_{1}+\prod_{1<j \neq 3}\left(a_{1}^{*} a_{j}^{*}+s_{1 j}^{j}\right)\right]\left[a_{1}+\prod_{1<j \neq 4}\left(a_{1}^{*} a_{j}^{*}+s_{1 j}^{j}\right)\right] \\
= & a_{1}+\left(a_{2}+\mathbf{s}_{1}\right)\left[a_{1} \prod_{3<j}\left(a_{1}^{*} a_{j}^{*}+s_{1 j}^{j}\right)+a_{3}+\mathbf{s}_{1}\right] \\
& \times\left[a_{1} \prod_{2<j \neq 4}\left(a_{1}^{*} a_{j}^{*}+s_{1 j}^{j}\right)+a_{4}+\mathbf{s}_{1}\right] \\
\leq & a_{1}+\left(a_{2}+\mathbf{s}_{1}\right)\left[\prod_{3<j} a_{1} s_{1 j}^{j}+a_{1}^{*} a_{2}^{*}+s_{12}^{2}\right]\left[\prod_{2<j \neq 4} a_{1} s_{1 j}^{j}+a_{1}^{*} a_{2}^{*}+s_{12}^{2}\right] \\
= & a_{1}+\left(a_{2}+\mathbf{s}_{1}\right)\left[a_{1}^{*} a_{2}^{*}+s_{12}^{2}+\prod_{3<j} a_{1} s_{1 j}^{j}\left(\prod_{2<j \neq 4} a_{1} s_{1 j}^{j}+a_{1}^{*} a_{2}^{*}+s_{12}^{2}\right)\right] \\
= & a_{1}+\left(a_{2}+\mathbf{s}_{1}\right)\left[a_{1}^{*} a_{2}^{*}+s_{12}^{2}+\prod_{3<j} a_{1} s_{1 j}^{j}\left(\prod_{2<j \neq 4} a_{1} s_{1 j}^{j}+a_{1} s_{12}^{2}\right)\right] \\
\leq & \left.\left.a_{1}+\prod_{2<j}\left(a_{1}^{*} a_{j}^{*}+s_{1 j}^{j}\right)\right] a_{1}^{*} a_{2}^{*}+s_{12}^{2}+\prod_{2<j} a_{a_{1}} s_{1 j}^{j}+a_{1} s_{12}^{2}\right] \\
\square & {\left[\mathbf{s}_{1}\right] }
\end{aligned}
$$

This short proof is due to Alan Day. $L$.

LEMMA 3.7. If $S \subseteq R_{\phi}$ is right uniserial then the $\tilde{s} a_{1}(s \in S)$ form a chain in

Proof. Consider the dual lattice $L^{*}$ with frame $\phi^{*}$. By Lemma 2.6 its coordinate ring is $R^{\mathrm{op}}$. Hence, $S^{\mathrm{op}} \subseteq R^{\mathrm{op}}$ and Lemma 2.4 establishes an order preserving map $S^{\mathrm{op}} r^{*} \mapsto r_{21}^{*} a_{2}^{*}+a_{1}^{*}$ of the lattice of left ideals of $S^{\text {op }}$ into $L^{*}$. In particular, the $r_{21}^{*} a_{2}^{*}+a_{1}^{*}=r_{12} a_{1}+a_{1}^{*}(r \in S)$ form a chain and so do the $r_{12} a_{1}=\tilde{r} a_{1}$.

LEMMA 3.8. If $n=3, L$ Arguesian, and $s^{k}$ invertible in $R_{\phi}$, then $\mathbf{s}_{\phi}=\mathbf{t}_{k}=$ $\left(a_{i}+\mathbf{s}_{i}\right)\left(a_{j}+\mathbf{s}_{j}\right)$, where $k \neq i \neq j \neq k$ and $t^{h}=\left(-1 / s^{k}\right) s^{h}$. 
Proof. By (2.4) we have $a_{h}+\mathbf{s}_{h}=a_{h}+\mathbf{t}_{h}$, whence $\mathbf{s}_{\phi}=\mathbf{t}_{\phi}$. Thus we may consider $k=1, s^{1}=-1$, only. Then by $(2.5)$ we get

$$
\begin{aligned}
{\left[a_{2}+\right.} & \left.\left(a_{3}+\ominus 1_{21}\right)\left(a_{1}+s_{23}^{3}\right)\right]\left[a_{3}+\left(a_{2}+\ominus 1_{31}\right)\left(a_{1}+s_{32}^{2}\right)\right] \\
& =\left[a_{2}+\left(a_{3}+\ominus 1_{12}\right)\left(a_{2}+s_{13}^{3}\right)\right]\left[a_{3}+\left(a_{2}+\ominus 1_{13}\right)\left(a_{3}+s_{12}^{2}\right)\right] \\
& =\left(a_{2}+s_{13}^{3}\right)\left(a_{3}+s_{12}^{2}\right)=\mathbf{s}_{1} .
\end{aligned}
$$

4. Representation of automorphisms. Returning to the proof of Theorem 2.7 we represent the elementary automorphisms $\alpha_{i j}^{r}$ and $\mu_{q i}$ of $S_{S}^{n}$ given by $\alpha_{i j}^{r} e_{i}=$ $e_{i}-r e_{j}, \mu_{q i} e_{i}=q^{-1} e_{i}$, and $\alpha_{i j}^{r}\left(e_{k}\right)=\mu_{q i}\left(e_{k}\right)=e_{k}$ for $k \neq i$. For this section we assume $L$ is freely generated by $\phi$ over $S \subseteq R_{\phi}$ maybe under additional relations of the form $\tilde{s} \leq \bar{t}$ with integer $s, t$.

THEOREM 4.1. For all $i \neq j$ and $r$ in $S$ there is an automorphism $\alpha_{i j \phi}^{r}$ of $L$ with fixpoints $a_{j}+\mathbf{s}_{k}$ and $\alpha_{i j \phi}^{r} \mathbf{s}_{k}=\left(\alpha_{i j}^{r} \mathbf{s}\right)_{k}$ for $\mathbf{s}$ in $S^{n}$ and $k \neq j$. Moreover, $\alpha_{12 \phi}^{r} s_{21}=(-s, 1+s r, 0, \ldots, 0)_{\phi}$ and $\bar{s}$ is a fixpoint if $s \in S$ with $s r \in R s$.

Proof. Let $i=1, j=2$. Consider $a_{1}^{\prime}=r_{12}, c_{12}^{\prime}=r \oplus 1_{12}, a_{k}^{\prime}=a_{k}$, and $c_{k l}^{\prime}=c_{k l}$ for $k, l>1$. By Lemma 2.1 these elements give rise to an $n$-frame $\phi^{\prime}$ of $L$. Fix $k, l>1$. We claim that the coordinate rings $R_{k l \phi}$ and $R_{k l \phi^{\prime}}$ coincide. This is obvious for the underlying sets, zero, and unit. For $n \geq 4$ we use the fact that addition and multiplication can be defined without the index 1 . Now, let $n=3$. The spanning 3-diamonds $(x, y, z, t)$ and $(\bar{x}, \bar{y}, z, t)$, with $z=a_{3}, t=c_{32}, x=c_{12}$, $y=a_{1}, \bar{x}=c_{12}^{\prime}, \bar{y}=a_{1}^{\prime}$, yield the same coordinate domain $D=R_{32 \phi}=R_{32 \phi^{\prime}}$ in the Day-Pickering construction [8]. Moreover, the hypotheses of Lemmas 3.4 and 3.7 in $[8]$ are satisfied, which means both 3 -diamonds induce the same ring structure on $D$ in the Day-Pickering version. In view of Lemmas 3.5 and 3.14 of [8] this carries over to the von Neumann version. Similarly, for $R_{23 \phi}$ and $R_{23 \phi^{\prime}}$ consider $z=a_{2}, t=c_{32}, x=c_{13}, y=a_{1}, \bar{x}=c_{13}^{\prime}$, and $\bar{y}=a_{1}^{\prime}$. Since

$$
\begin{aligned}
c_{13}^{\prime} & =\left(c_{12}^{\prime}+c_{23}^{\prime}\right)\left(a_{1}^{\prime}+a_{3}^{\prime}\right)=\left(r \oplus 1_{12}+c_{23}\right)\left(r_{12}+a_{3}\right) \\
& =\left[\left(r_{12}+a_{3}\right)\left(c_{13}+a_{2}\right)+c_{23}\right]\left(r_{12}+a_{3}\right)=\left(r_{12}+a_{3}\right)\left(c_{13}+a_{2}\right),
\end{aligned}
$$

by definition of addition the independence results for the ring structure given in $\mathrm{A}$. Day [5, Part III] apply.

Also for $n=3$, associating with $\phi$ and $\phi^{\prime}$ dual 3-frames $\phi^{*}$ and $\phi^{*}$ as in Lemma 2.6 we have the coordinate rings $R_{13 \phi^{*}}$ and $R_{13 \phi^{\prime *}}$ coincide. Namely, $c_{13}^{\prime *}=c_{13}+$ $a_{2}=c_{13}^{*}, a_{1}^{\prime *}=a_{2}+a_{3}=a_{1}^{*}, a_{3}^{\prime *}=r_{12}+a_{2}=a_{1}+a_{2}=a_{3}^{*}, a_{2}^{\prime *}=r_{21}^{*}$, and $c_{21}^{\prime *}=r \oplus 1_{21}^{*}$. Thus, the claim follows from the above by duality and symmetry.

Finally, $\tilde{s}_{\phi^{\prime}} \leq \bar{t}_{\phi^{\prime}}$ for each of the additional relations $\tilde{s} \leq \bar{t}$. This is a consequence of $(3.9)$ and $\tilde{s}_{23 \phi^{\prime}}=s_{23} a_{3} \leq\left(t_{32}+a_{3}\right) a_{2}=\left(t_{32 \phi^{\prime}}+a_{3}^{\prime}\right) a_{2}^{\prime}$.

Now, in view of Theorem 2.2 (and (2.6)) $L$ is freely generated by $\phi$ and the $s_{k l \phi}$ (and $s_{13 \phi}^{*}$ if $n=3$ ), with $s \in S, k, l>1$, subject to the frame relations, the relations of the rings $S_{k l \phi}$ (and $S_{13 \phi^{*}}$ ), the relations given by the canonical isomorphisms between them, and the additional relations. These relations are satisfied by $\phi^{\prime}$ and $s_{k l \phi}=s_{k l \phi^{\prime}}$ (and $s_{13 \phi}^{*}=s_{13 \phi^{\prime}}^{*}$ ) too, as we just showed. Hence there is an endomorphism $\alpha=a_{12 \phi}^{r}$ of $L$ mapping $\phi$ onto $\phi^{\prime}$ and fixing the $s_{k l \phi}$ (and $s_{13 \phi}^{*}$ if $n=3)$ for $s$ in $S$ and $k, l>1$. In particular, $\alpha a_{1}=r_{12}$ and $\alpha c_{12}=r \oplus 1_{12}$.

We claim that $a_{2}+s_{k 1}$ is a fixpoint for $k>2$. For $n=3$ this is so, since

$$
s_{13}^{*}=\left(s_{21}^{*} c_{13}^{*}+a_{2}^{*} a_{3}^{*}\right) c_{12}^{*}+a_{1}^{*} a_{3}^{*}=\left(\ominus s_{32}+a_{1}\right)\left(c_{12}+a_{3}\right)+a_{2}=s_{31}+a_{2},
$$


using (2.5) twice. For $n \geq 4$ observe that the automorphism $\alpha_{12 h}^{r}, h \neq k$, of Lemma 2.5 coincides with $\alpha$ on the sublattice generated by $a_{1}, c_{12}, a_{2}$, and the $s_{k 2}(s \in S)$ since it does so on the generators. It follows that

$$
\alpha \mathbf{s}_{k}=\left(a_{1}^{*} a_{k}^{*}+s_{k 1}^{1}\right)\left(r_{12}+a_{1}^{*} a_{2}^{*} a_{k}^{*}+s_{k 2}^{2}\right) \prod_{2<j \neq k}\left(a_{j} a_{k}+s_{k j}^{j}\right)=(\alpha \mathbf{s})_{k}
$$

for $k>2$ since

$$
\begin{aligned}
a_{1}^{*} a_{2}^{*} a_{k}^{*}+\left(a_{2}+s_{k 1}^{1}\right)\left(r_{12}+s_{k 2}^{2}\right) & =a_{1}^{*} a_{2}^{*} a_{k}^{*}+\left(a_{2}+s_{k 1}^{1}\right)\left(a_{1}+s^{2} \ominus s^{1} \otimes r_{k 2}\right) \\
& =\left(a_{1}^{*} a_{k}^{*}+s_{k 1}^{1}\right)\left(a_{2}^{*} a_{k}^{*}+s^{2} \ominus s^{1} \otimes r_{k 2}\right)
\end{aligned}
$$

by (2.5). In particular, $a_{2}+\mathbf{s}_{k}$ is a fixpoint. From $s_{12}=\left(a_{1}+a_{2}\right)\left(c_{1 k}+s_{k 2}\right)$ one derives

$$
\begin{aligned}
\alpha s_{12} & =\left(a_{1}+a_{2}\right)\left[\left(a_{2}+c_{k 1}\right)\left(a_{1}+\ominus r_{k 2}\right)+s_{k 2}\right] \\
& =\left(a_{1}+a_{2}\right)\left(s \oplus r_{k 2}+c_{k 1}\right)=s \oplus r_{12}
\end{aligned}
$$

by (2.3). Since $\phi \cup S_{12}$ generates $L$, it is clear now that $\alpha_{12 \phi}^{-r}$ is the inverse automorphism. From $s_{1 k}=\left(s_{12}+c_{2 k}\right)\left(a_{1}+a_{k}\right)$ one gets

$$
\alpha s_{1 k}=\left(s \oplus r_{12}+c_{2 k}\right)\left(r_{12}+a_{k}\right)=\left(a_{2}+s_{1 k}\right)\left(r_{12}+a_{k}\right)
$$

by (2.2) and (2.5). It follows that $a_{2}+s_{1 k}$ is a fixpoint,

$$
\alpha \mathbf{s}_{1}=\left(a_{1}^{*} a_{2}^{*}+s_{12}^{2}+r_{12}\right) \prod_{k>2}\left(a_{1}^{*} a_{k}^{*}+s_{1 k}^{k}\right)=(\alpha \mathbf{s})_{1},
$$

and $a_{2}+\mathbf{s}_{1}$ is a fixpoint too.

Obviously, the $\bar{s} a_{i}=a_{i}\left(a_{j}+s_{j i}\right)$ are fixed under $\alpha$ for $s$ in $S, i \neq 1$. If $s r \in R s$ we derive with Lemma 2.4,

$$
\begin{aligned}
\alpha\left(\bar{s} a_{1}+\bar{s} a_{2}\right) & =\alpha\left(\left(a_{1}+a_{2}\right)\left(a_{3}+s_{31}\right)\right)+\bar{s} a_{2} \\
& =\left(a_{1}+a_{2}\right)\left[a_{3}+\left(a_{1}+s \otimes r_{32}\right)\left(a_{2}+s_{31}\right)\right]+\left(a_{1}+a_{2}\right)\left(a_{3}+s_{32}\right) \\
& =\bar{s} a_{1}+s \bar{a}_{2} .
\end{aligned}
$$

Hence, $\bar{s}=\sum \bar{s} a_{i}$ is a fixpoint.

Finally, using (2.5) twice one has

$$
\begin{aligned}
a_{3}+s_{21} & =\left(a_{1}+c_{32}\right)\left(a_{3}+s_{21}\right)+a_{3}=a_{3}+\left(a_{1}+c_{32}\right)\left(a_{2}+\ominus s_{31}\right) \\
& =a_{3}+\left(a_{2}+\ominus s_{31}\right)\left(\ominus r_{12}+1 \oplus s \otimes r_{32}\right),
\end{aligned}
$$

whence

$$
\begin{aligned}
\alpha s_{21} & =\alpha\left(\left(a_{1}+a_{2}\right)\left(a_{3}+s_{21}\right)\right) \\
& =\left(a_{1}+a_{2}\right)\left[a_{3}+\left(a_{2}+\ominus s_{31}\right)\left(a_{1}+1 \oplus s \otimes r_{32}\right)\right] .
\end{aligned}
$$

THEOREM 4.2. For all $i$ and invertible $q$ in $S \subseteq R_{\phi}$ there is an automorphism $\mu_{q i \phi}$ of $L$ with $\mu_{q i \phi} s_{i k}=q \otimes s_{i k}, \mu_{q i \phi} \mathbf{s}_{k}=\left(\mu_{q i} \mathbf{s}\right)_{k}$ for $k \neq i$. Moreover, if $n=3$ and $L$ Arguesian then $a_{i}+\mathbf{s}_{i}$ is a fixpoint and $\mu_{q i \phi} \mathbf{s}_{\phi}=\left(\mu_{q i} \mathbf{s}\right)_{\phi}$.

PROOF. Let $i=1$. As in the proof of Theorem 4.1 consider a new frame $\phi^{\prime}$ with $a_{i}^{\prime}=a_{i}, c_{12}^{\prime}=q_{12}$, and $c_{k l}^{\prime}=c_{k l}$ for $k, l>1$. Indeed, by $(2.1)$ this yields a frame and, as before, $R_{k l \phi}$ coincides with $R_{k l \phi^{\prime}}$ for $k, l>1$ and the relations $\tilde{s} \leq \bar{t}$ are transferred from $\phi$ to $\phi^{\prime}$. Hence, there exists an endomorphism $\mu=\mu_{q i \phi}$ of $L$ mapping $\phi$ onto $\phi^{\prime}$ and fixing the $s_{k l}$ for $s$ in $S, k, l>1$. 
For $k>2$ and $s$ in $S$ one has

$$
\mu s_{1 k}=\mu\left(\left(c_{12}+s_{2 k}\right)\left(a_{1}+a_{k}\right)\right)=\left(q_{12}+s_{2 k}\right)\left(a_{1}+a_{3}\right)=q \otimes s_{1 k}
$$

and

$$
\mu s_{12}=\mu\left(\left(s_{13}+c_{32}\right)\left(a_{1}+a_{2}\right)\right)=\left(q \otimes s_{13}+c_{32}\right)\left(a_{1}+a_{2}\right)=q \otimes s_{12} .
$$

Thus, $\mu_{1 / q 1 \phi}$ is the inverse of $\mu$. Moreover, by (2.1)

$$
\begin{aligned}
\mu s_{k 1} & =\mu\left(\left(s_{k l}+c_{1 l}\right)\left(a_{1}+a_{k}\right)\right)=\left(s_{k l}+q_{1 l}\right)\left(a_{1}+a_{k}\right) \\
& =\left(s_{k l}+1 / q_{l 1}\right)\left(a_{1}+a_{k}\right)=s / q_{k 1} .
\end{aligned}
$$

Hence, $\mu \mathbf{s}_{k}=(\mu \mathbf{s})_{k}$ for $k \neq 1$. Finally, if $n=3$ then $a_{1}+\mathbf{s}_{1}$ is a fixpoint by $(2.4)$ and one concludes that $\mu \mathbf{s}_{\phi}=(\mu \mathbf{s})_{\phi}$.

PROOF OF THEOREM 2.7. If $n \leq 4$ then by Lemmas 3.5 and 3.6 one has $\mathbf{s}_{\phi}=\left(a_{i}+a_{j}+\mathbf{s}_{i}\right) \prod_{k \neq i, j}\left(a_{k}+\mathbf{s}_{k}\right)$ for all $\mathbf{s}$ in $S^{n}$, whence $\alpha_{i j \phi}^{r} \mathbf{s}=\left(\alpha_{i j}^{r} \mathbf{s}\right)_{\phi}$ and $\mu_{q i \phi} \mathbf{s}_{\phi}=\left(\mu_{q i} \mathbf{s}\right)_{\phi}$ by Theorems 4.1 and 4.2. Let $n=3$ and $L$ Arguesian. One has $\mathbf{s}_{\phi}=\bar{q} \mathbf{t}_{k}$, choosing $k$ such that $s^{k}=-q, t^{k}=-1$, and $s^{i}=q t^{i}$ for all $i$-which is possible since $S$ is uniserial. Namely, by (3.10) $\mathbf{s}_{\phi} \leq \bar{q}$, and by (2.4) $a_{i}+\mathbf{s}_{i} \leq a_{i}+\mathbf{t}_{i}$. Thus in view of Lemma 3.8 it suffices to show $\bar{q}\left(a_{j}+\mathbf{s}_{j}\right)=\bar{q}\left(a_{j}+\mathbf{t}_{j}\right)$ for $j \neq k$. By Theorem 4.1 the transformation $\alpha_{k i \phi}^{-t^{i}}(i \neq j, k)$ reduces this to

$$
\bar{q}\left(a_{j}+\ominus q_{j k}\right)=\bar{q}\left(a_{j}+s_{j k}^{k}\right)=\bar{q}\left(a_{j}+t_{j k}^{k}\right)=\bar{q}\left(a_{j}+a_{k}\right),
$$

which is valid, obviously, by (3.8).

Again, consider $\alpha=\alpha_{12}^{r}$ only. By Theorrem $4.1 \alpha \bar{q}=\bar{q}$ and, for $k \neq 2$, $\alpha \mathbf{s}_{\phi}=\bar{q} \alpha \mathbf{t}_{k}=\bar{q}(\alpha \mathbf{t})_{k}=(\alpha \mathbf{s})_{\phi}$. Now, assume $k=2$ is the only possible choice, in particular, $t^{1}$ is not invertible. Since $S$ is local $1+t^{1} r$ has an inverse $u$. Application of $\alpha_{23 \phi}^{t^{3}}$ to the instance $\left(a_{3}+u \otimes t_{21}^{1}\right)\left(a_{2}+r \otimes t_{13}^{3}\right)=\left(a_{3}+u \otimes t_{21}^{1}\right)\left(a_{1}+u \otimes t^{3} \ominus t_{23}^{3}\right)$ of (2.5) yields

$$
\left(a_{3}+u \otimes t_{21}^{1}\right)\left(r_{12}+t_{23}^{3}\right)=\left(a_{3}+u \otimes t_{21}^{1}\right)\left(a_{1}+u \otimes t_{23}^{3}\right)
$$

since

$$
\alpha_{23 \phi}^{-t^{3}}\left(r_{12}+t_{23}^{3}\right)=\left(a_{2}+r \otimes t_{13}^{3}\right)\left(a_{3}+r_{12}\right)+a_{2}=a_{2}+r \otimes t_{13}^{3}
$$

by Theorem 4.1. It follows that

$$
\alpha \mathbf{t}_{2}=\left[a_{3}+\left(a_{2}+\ominus t_{31}^{1}\right)\left(a_{1}+u_{23}\right)\right]\left(r_{12}+t_{23}^{3}\right)=(u \mathbf{t})_{2}
$$

with (2.1) and (2.5). Consequently, $\alpha \mathbf{s}_{\phi}=\bar{q}\left(u t^{1},-1, u t^{3}\right)_{2}=(\alpha \mathbf{s})_{\phi}$. The $\mu$ 's have already been dealt with in Theorem 4.2 .

Finally, we have completed the proofs for the claims of $\S 2$. In particular, from Corollaries 2.10, 3.4 and Lemma 3.5 we get

COROLLARY 4.3. Let $L$ be generated by an $n$-frame of characteristic $m \neq 0$, e.g. by a reduced frame $\phi_{m}$. Then $L$ is a subdirect product of lattices $L\left(Z_{p^{k}}^{n}\right)$ with $p^{k}$ dividing $m, p$ prime, and the image of the generating frame being canonical.

THEOREM 4.4. Let $L$ be freely generated by an $n$-frame $\phi$ subject to all relations $\tilde{s} \leq \bar{t}, s, t>0$ integers. Then $Z$ is a subring of $R_{\phi}$ and there is a homomorphism $\psi \mapsto \psi_{\phi}$ of the automorphism group $\operatorname{GL}(n, Z)$ of $Z^{n}$ into that of $L$ such that $\psi_{\phi} \mathbf{s}_{\phi}=$ 
$(\psi \mathbf{s})_{\phi}$ for each $\mathbf{s}$ in $Z^{n}$ with $s^{1}, \ldots, s^{n}$ relatively prime. Moreover, all $\tilde{s}$ and $\bar{s}$ $(s \in Z)$ are fixpoints of $\psi_{\phi}$.

Proof. Of course, $R_{\phi}$ has characteristic 0 and contains $Z$ since $L\left({ }_{Q} Q^{n}\right)$ is a homomorphic image of $L$. Again, it suffices to consider the elementary maps $\mu_{q i}$ and $\alpha_{i j}^{r}$. Here, $q= \pm 1$ and because of symmetry and the fact that $\alpha_{i j}^{r}$ is a power of $\alpha_{i j}^{1}$ we have to deal with $\alpha=\alpha_{12}^{1}$ only. That $\tilde{s}$ and $\bar{s}$ are fixpoints of $\mu_{-1 i}$ is obvious by (3.5) and (3.8). Also $\alpha \bar{s}=\bar{s}$ by Theorem 4.1 and $\alpha\left(\tilde{s} a_{i}\right)=\alpha\left(s_{i j} a_{i}\right)=\tilde{s} a_{i}$ for $i \neq 1$. Since $\alpha\left(\tilde{s} a_{1}\right)=\alpha\left(a_{1}\left(\tilde{s} a_{2}+\ominus 1_{12}\right)\right)=c_{12}\left(\tilde{s} a_{2}+a_{1}\right)=\tilde{s} c_{12}$ by (3.2) and (3.3), we get $\alpha \tilde{s}=\tilde{s}$ by $(3.1)$.

The case $n \geq 4$ is settled by Theorems 4.1 and 4.2 if we can apply Lemma 3.6, i.e. if the $\tilde{s} a_{1}(s \in Z, s \neq 0)$ generate a distributive sublattice of $L$. Indeed, by (3.5) any finite collection belongs to the interval $\left[0, \tilde{u} a_{1}\right]$ of the sublattice generated by $\phi_{u}, u \neq 0$ a common multiple of the associated integers, and this interval is distributive in view of Corollary 4.3 .

Now let us deal with $n=3$ and $L$ Arguesian. Theorem 4.2 tells all about $\mu_{q i}$. Recall that, e.g., $(-1, s, t)=(1,-s,-t)=\left(a_{3}+s_{12}\right)\left(a_{2}+t_{13}\right),(0, s, t)=$ $\left(a_{2}+a_{3}\right)\left(a_{1}+(-1, s, t)\right)$, and $a_{1}+(0, s, t)=a_{1}+(-1, s, t)$ by Lemma 3.8 and Proposition 2.3-we omit the subscript $\phi$ where no confusion is possible. Now, for fixed $r, s$ and $t$ in $Z$ let $A=c_{12}+(-1, s+1, t), B=a_{2}+(r, 0, t), C=a_{3}+(r, s-r, 0)$, and $D=a_{1}+(0, s-r, t)$. Then $(\alpha(r, s, t))_{\phi}=D B C$. On the other hand we show $\alpha B=B$ whence $\alpha(r, s, t)_{\phi}=A B C$ by Theorem 4.1. Thus, we have to show $A B C=D B C$ for $r, s, t$ relatively prime. We break the calculation into a series of steps.

$$
\alpha B=B
$$

Proof. Let $X=\left(c_{12}+t_{23}\right)\left(a_{3}+(r,-r-1,0)\right)$. By Theorem 4.1 one has $\alpha B=a_{2}+X$. The inequality $X \leq B$ is the CP-statement for the normal triangles $a_{3}, t_{23},(r,-1, t)$ and $(r,-r-1,-1), c_{12}, a_{2}$. Since $L$ is Arguesian it suffices to derive the AP-statement, which is a consequence of $Y \leq a_{1}+\left(a_{3}+r_{21}\right)\left(a_{2}+r_{31}\right)$, where $Y=\left(a_{2}+a_{3}\right)\left(c_{12}+(r,-r-1,-1)\right)$. Since $\alpha_{32}^{r+1} Y=r_{32}$ the latter is obtained from $a_{3} \leq a_{1}+r_{31} \leq a_{1}+\left(c_{32}+r_{21}\right)\left(a_{2}+r_{31}\right)$ by the transformation $\alpha_{32}^{1}$.

Consequently $a_{2}+X \leq B$. Equality follows since both sides are complements of $a_{2}+a_{3}$ in $\left[\left(a_{2}+a_{3}\right) B, a_{2}+a_{3}+r_{21}\right]$. Indeed, Proposition 2.3 yields $a_{2}+a_{3}+X=$ $a_{2}+a_{3}+r_{21}$, and by (3.3) one has

$$
\left(a_{2}+a_{3}\right) B=a_{2}+t_{23}\left(a_{3}+r_{21}\left(a_{2}+a_{3}\right)\right)=a_{2}+t_{23 \phi_{r}} .
$$

By Corollary 4.3, evaluating $X$ over $\phi_{r}$ we have $(r,-r-1,0)_{\phi_{r}}=a_{2 \phi_{r}}$, whence $X_{\phi_{r}} \geq t_{23 \phi_{r}}$ and $a_{2}+t_{23 \phi_{r}} \leq a_{2}+X$.

$$
\left(a_{i}+s_{k j}\right)\left(a_{k}+s_{i j}\right)=\left(\ominus 1_{i k}+a_{j}\right)\left(a_{k}+s_{i j}\right)+\tilde{s} a_{k} .
$$

ProOF. Let $i=1, j=2, k=3$. Both sides of the identity are complements of $a_{3}$ in $\left[\tilde{s} a_{3}, a_{3}+s_{12}\right]$. Hence by $(2.5)$ it suffices to show $a_{1}+s_{32} \geq\left(s \ominus 1_{13}+c_{23}\right)\left(a_{3}+s_{12}\right)$. This is CP for the normal triangles $s \ominus 1_{13}, a_{3}, s_{32}$ and $c_{23}, s_{12}, a_{1}$. We have to prove $\mathrm{AP}$ which is $s_{13} \leq \bar{s} a_{2}+\left(s_{32}+s \ominus 1_{13}\right)\left(a_{1}+c_{23}\right)$ and reduces to $a_{1} \leq X$ with $X=\bar{s} a_{2}+\left(s_{32}+\ominus 1_{13}\right)\left(\ominus s_{13}+c_{23}\right)$ via the transformation $\alpha_{13}^{-s}$. But, by $(2.2)$ and (3.8),

$$
X=\bar{s} a_{2}+\left(\Theta s_{12}+\Theta 1_{13}\right)\left(\Theta s_{12}+\varsigma_{23}\right) \geq\left(a_{1}+\ominus s_{12}\right) a_{2}+\Theta s_{12} \geq a_{1} .
$$




$$
A C \leq D+\tilde{s} t_{13}
$$

PROOF. This is CP for the normal triangles $c_{12},(r, s-r,-1), a_{1}$ and $(-1, s+$ $1, t), a_{3},(-1, s-r, t)+\tilde{s} t_{13}$. AP is implied by $X \leq Y$, where

$$
X=\left(c_{12}+(r, s-r,-1)\right)\left(a_{3}+s \oplus 1_{12}\right)
$$

and

$$
Y=\left(a_{1}+s \ominus r_{32}\right)\left(a_{3}+s \ominus r_{12}\right)+\tilde{s} a_{1}+a_{2}
$$

- since $a_{3}+\tilde{s} t_{13}=a_{3}+\tilde{s} a_{1}$ by (3.1). Now, by Theorem 4.1

$$
\alpha^{-1} X=\left(a_{1}+(r, s,-1)\right)\left(a_{3}+s_{12}\right)=\left(a_{1}+s_{32}\right)\left(a_{3}+s_{12}\right),
$$

whence $X=(-1, s+1,-1)+\tilde{s} a_{1}$ by (4.2). Thus, by (4.2), Lemma 3.1, and (2.1) $Y \geq \ominus 1_{13}+a_{2}+\tilde{s} a_{1} \geq X$.

$$
\left(a_{1}+(0, s+1, t)\right)\left(a_{3}+(-r-2, s+1,0)\right) \leq c_{12}+a_{1} \widetilde{s+1}+(-1, s-r, t) .
$$

PROOF. This is CP for the normal triangles $a_{1},(-r-2, s+1,-1), c_{12}$ and $(-1, s+1, t), a_{3},(-1, s-r, t)+a_{2} \widetilde{s+1}-$ use (3.1). AP is a consequence of $X \leq Y+Z$, where $X=(-1, s+1,-1), Y=\left(a_{1}+a_{2}\right)((-1, s+1, t)+(-1, s-r, t))$, and $Z=\left(a_{3}+s \ominus r_{12}\right)\left(c_{12}+(-r-2, s+1,-1)\right)$. Now, by Theorems 4.1 and $(4.2)$

$$
\begin{aligned}
\alpha^{-1} Z & =\left(a_{3}+s \ominus r \ominus 1_{12}\right)\left(a_{1}+(-r-2, s-r-1,-1)\right) \\
& =\left(a_{3}+s \ominus r \ominus 1_{12}\right)\left(a_{1}+s \ominus r \ominus 1_{32}\right) \\
& =(-1, s-r-1,-1)+a_{3}(s-r-1)^{\sim} .
\end{aligned}
$$

Therefore, $\alpha^{-1-s}$ transforms $X \leq Y+Z$ into

$$
\ominus 1_{13} \leq\left(a_{1}+a_{2}\right)\left(t_{13}+(-1,-r-1, t)\right)+(-1,-r-1,-1)+a_{3}(s-r-1)^{\sim},
$$

which is an easy consequence of modularity.

$$
\begin{aligned}
\left(a_{1}+a_{2}\right) & (-1, s+1, t)+(r,-1, t)) \\
\leq & \left(a_{1}+a_{2}\right)((-1,-1,-1)+(-r-2, s+1,-1))+s \oplus 1_{12} \tilde{t} .
\end{aligned}
$$

PROOF. In view of (2.3) this is a consequence of CP for the normal triangles $a_{2},(-1, s+1, t),(-1, s+1,-1)+s \oplus 1_{12} \tilde{t}$ and $a_{1},(r,-1, t),(r,-1,-1)$. Due to the fact that $a_{2}+s \oplus 1_{12} \tilde{t}=a_{2}+\tilde{t} a_{1}$ by (3.1), and due to (4.2), AP follows from $(-1,-1, t)+\tilde{t} a_{1} \leq(-1,-1,-1)+\tilde{t} a_{1}+X$, where

$$
X=\left(a_{3}+s \oplus 1_{12}\right)\left((-1, s+1,-1)+a_{2}+t_{13}\right)\left(a_{3}+r_{21}\right)\left((r,-1,-1)+a_{1}+t_{23}\right) .
$$

Now $X \geq Y=a_{3}\left(a_{2}+\ominus 1_{13}+t_{13}\right)\left(a_{1}+\ominus 1_{23}+t_{23}\right)$ and the transformation $\alpha_{21}^{+1}$ maps $(-1,-1,-1)+Y$ onto

$$
\begin{aligned}
& \ominus \mathbf{1}_{23}+a_{3}\left(c_{12}+\ominus \mathbf{1}_{13}+t_{13}\right)\left(a_{1}+\ominus \mathbf{1}_{23}+t_{23}\right) \\
& =\left(a_{3}+\ominus \mathbf{1}_{23}\right)\left(c_{12}+\ominus \mathbf{1}_{23}+t_{23}\right)\left(a_{1}+\ominus \mathbf{1}_{23}+t_{23}\right) \geq t_{23},
\end{aligned}
$$

the image of $(-1,-1, t)$ - use (2.1) and (2.2)!

$$
A B \leq D+F+a_{2}(t s+t)^{\sim} \text { for } F=\left(a_{1}+t_{23}\right)(-1, s+1, t) .
$$

PROOF. This is the CP-statement for the normal triangles $c_{12}, a_{2},(-1, s-r, t)+$ $c_{12}(t s+t)^{\sim}$ and $(-1, s+1, t),(r,-1, t), a_{1}+F$. By (4.5) and (4.4) the AP-statement is a consequence of

$$
\left(x_{0}+x_{1}\right)\left(y_{0}+y_{1}\right)+s \oplus 1_{12} \tilde{t} \leq\left(a_{2}+t_{13}\right)\left(a_{1}+t_{23}\right)+\left(x_{0}+x_{2}\right)\left(y_{2}+y_{0}\right) .
$$


where $x_{0}=a_{1}, x_{1}=a_{2}, x_{2}=(-1, s+1, t), y_{0}=(-r-2, s+1,-1), y_{1}=$ $(-1,-1,-1)$, and $y_{2}=a_{3}$. Now,

$$
\begin{aligned}
\left(a_{2}+t_{13}\right)\left(a_{1}+t_{23}\right) & =(-1,-1, t)+\tilde{t} a_{1}+\tilde{t} a_{2} \\
& =\left(y_{1}+y_{2}\right)\left(x_{1}+x_{2}\right)+\tilde{t}\left(a_{1}+a_{2}\right)
\end{aligned}
$$

by (4.2). Hence, it suffices to verify that $\mathbf{x}$ and $\mathbf{y}$ are axially perspective. But, the CP-statement for $\mathbf{x}$ and $\mathbf{y}$ is $\left(a_{1}+s \oplus 1_{32}\right)\left(a_{2}+\ominus 1_{13}\right) \leq a_{3}+s \oplus 1_{12}$, a consequence of $(2.5)$.

$$
\tilde{u}\left(a_{i}+\mathbf{s}_{i}\right) \leq \tilde{v} a_{i}+\mathbf{s}_{i \phi_{v}} \text { for } u s^{1} s^{2} s^{3} \text { dividing } v .
$$

ProOF. Let $i=1, \mathrm{~s}=(r, s, t)$. By $(3.5)$ we have $a_{1}\left(\ominus s_{12}+u_{23} a_{2}\right) \leq a_{1} \widetilde{-s u} \leq \tilde{v}$. Application of $\alpha^{s}$ yields $s_{12}\left(a_{1}+a_{3}+\tilde{u}\right)=s_{12}\left(a_{1}+u_{23} a_{2}\right) \leq \tilde{v}$ since $\tilde{v}$ is a fixpoint. Similarly, $t_{13}\left(a_{1}+a_{2}+\tilde{u}\right) \leq \tilde{v}$. Therefore one has

$$
\begin{aligned}
\tilde{u}\left(a_{1}+(-1, s, t)\right) & \leq \tilde{v}\left[a_{1}+\left(a_{3}+\tilde{v} s_{12}\right)\left(a_{2}+\tilde{v} t_{13}\right)\right] \\
& =\tilde{v}\left[a_{1}+\left(\tilde{v} a_{3}+\tilde{v} s_{12}\right)\left(\tilde{v} a_{2}+\tilde{v} t_{13}\right)\right]=\tilde{v} a_{1}+(-1, s, t)_{\phi_{v}} .
\end{aligned}
$$

$$
F \leq D+\tilde{v} a_{2} \text { for } v=t t(s+1)(s+2) \neq 0 .
$$

ProOF. By (4.2) we have

$$
\begin{aligned}
F & =\left(a_{2}+t_{13}\right)\left(a_{3}+\ominus 1_{12}+\tilde{t} a_{2}\right)\left(a_{3}+s \oplus 1_{12}\right) \\
& =\left(a_{2}+t_{13}\right)\left(a_{3}+\left(\ominus 1_{12}+\tilde{t} a_{2}\right) s \oplus 1_{12}\right) .
\end{aligned}
$$

For $u=t(s+2)$ it follows that

$$
\begin{aligned}
\alpha F & =\left(a_{2}+t_{13}\right)\left(a_{3}+\left(a_{1}+\tilde{t} a_{2}\right) s \oplus 2_{12}\right) \leq\left(a_{2}+t_{13}\right)\left(a_{3}+\tilde{u} a_{1}+\tilde{t} a_{2}\right) \\
& =t_{13}\left(a_{3}+\tilde{u} a_{1}\right)+\tilde{t} a_{2}=\tilde{u} t_{13}+\tilde{t} a_{2} \leq \tilde{u}
\end{aligned}
$$

by (3.7), (3.8), and (3.5). Consequently, by (4.7), $F=F_{\phi_{v}}$ and by Corollary 4.3 it suffices to verify $F \leq D+\tilde{v} a_{2}$ in the lattices $L\left(Z_{p^{k}}^{n}\right), p^{k}$ dividing $v$. But in such,

$$
\begin{gathered}
D+\tilde{v} a_{2}=D+a_{2}=a_{1}+a_{2}+t_{13} \geq F . \\
B C \leq D+\tilde{r} a_{3}+G \text { for } G=\left(a_{2}+t_{13}\right)(r, s-r,-1) .
\end{gathered}
$$

ProOF. This is CP for the normal triangles $a_{2},(r, s-r,-1),(-1, s-r, t)+G$ and $(r,-1, t), a_{3}, a_{1}+\tilde{r} a_{3}$. Now, $\left(a_{2}+t_{13}\right)\left(a_{1}+\tilde{r} a_{3}+t_{23}\right) \geq \tilde{r} a_{2}$ by $(3.1)$. Hence, in view of (4.2), AP for these triangles is a consequence of AP for $(r, s-r,-1), a_{2},(-1, s-$ $r, t)$ and $a_{1},(-1,-1,-1), a_{3}$. But, here CP is $\left(a_{1}+s \ominus r_{32}\right)\left(a_{2}+\ominus 1_{13}\right) \leq a_{3}+s \ominus r_{12}$, which follows from (2.5).

$$
G \leq D+\tilde{u} a_{3} \quad \text { for } u=r t-1 .
$$

Proof. By Theorem 4.1 we have

$$
\alpha_{13}^{-t}\left(r_{31} t_{13}\right)=a_{1}(r, 0, u)=a_{1}\left(a_{2}+r_{21}\left(\tilde{u} a_{2}+a_{1}\right)\right) \leq \tilde{u} a_{1},
$$

whence $G \leq\left(a_{2}+\tilde{u}\left(a_{1}+a_{3}\right)\right)\left(a_{1}+s \ominus r_{32}\right) \leq \tilde{u}$. It follows that

$$
G \leq \tilde{u} a_{1}+\tilde{u} s \ominus r_{32} \leq D_{\phi_{u}}+\tilde{u} a_{3} \leq D+\tilde{u} a_{3} .
$$

$$
\alpha(r, s, t) \leq(r, s-r, t) \quad \text { implies } \quad \alpha(-r,-s, t) \leq(-r, r-s, t) .
$$


ProOF. One has $\alpha \mu_{-13}=\mu_{-13} \alpha$, since by Theorems 4.1 and 4.2 this is the case on a generating subset of $\phi$. Therefore, one gets with Lemma 3.8

$$
\alpha(-r,-s, t)=\alpha \mu_{-13}(r, s, t)=\mu_{-13} \alpha(r, s, t) \leq \mu_{-13}(r, s-r, t)=(-r, r-s, t) .
$$

$$
\alpha(r,-s, t) \leq(r,-s-r, t) \quad \text { implies } \quad \alpha^{-1}(r, s, t) \leq(r, s+r, t) .
$$

ProOF. Due to Theorem 4.1 and (4.1) the first inequality is

$$
\left(c_{12}+(-1,1-s, t)\right) B\left(a_{3}+(r,-s-r, 0)\right) \leq(r,-s-r, t)
$$

and the second is

$$
\left(\ominus \mathbf{1}_{12}+(-1, s-1, t)\right) B\left(a_{3}+(r, s+r, 0)\right) \leq(r, s+r, t) .
$$

The automorphism $\mu_{-12}$ from Theorem 4.2 transforms one into the other.

$$
\alpha_{i j \phi}^{e}\left(\mathbf{s}_{\phi}\right) \leq\left(\alpha_{i j}^{e} \mathbf{s}\right)_{\phi}
$$

for $e= \pm 1$ and relatively prime $s^{1}, s^{2}, s^{3}$ with (a) $s^{j}$ odd, (b) $s^{k}$ even, or (c) $s^{j} \equiv 0$ $\bmod 4$.

Proof. Because of symmetry and (4.12) it suffices to consider $i=1, j=2$, and $e=1$. Let $\mathrm{s}=(r, s, t)$. By Theorem 4.1 and (4.1) one has $\alpha(r, s, t)=A B C$ and $(r, s-r, t)=D B C$. For $t=0, \pm 1$ the claim follows immediately from Proposition 2.3 and Theorem 4.1. If $r=0$ then $B \leq a_{2}+a_{3}$ and $A B \leq D$ is a consequence of (2.3). Thus, one may assume $t \neq 0, \pm 1, r \neq 0$, and $s \geq 0$-in view of (4.11). (4.3), (4.5), (4.9), and (4.10) jointly imply $A B C \leq D+X$, where

$$
X=\left(D+F+\tilde{q} a_{2}\right)\left(D+\tilde{s} a_{3}\right)\left(\tilde{u} a_{3}+\tilde{r} a_{3}\right)
$$

with $q=(s+1) t \neq 0$ and $u=r t-1 \neq 0$. It suffices to show $X \leq D$. Recall that $F \leq D+\tilde{v} a_{2}$ with $v=q t(s+2) \neq 0$ by (4.7). In particular, for $r=s$ it follows that $D+X \leq D+a_{2}\left(D+a_{3}\right) \leq D+a_{2}\left(a_{1}+a_{3}\right)=D$. Thus, assume $s-r \neq 0$. Then with (3.5) and (4.7) one derives $X \leq X_{\phi_{w}}$ for a suitable $w \neq 0$-observe that $X=\left(D+F+\tilde{q} a_{2}\right)\left(\tilde{u} a_{3}+\tilde{r} a_{3}\right)$ if $s=0$ ! Hence in view of Corollary 4.3 it suffices to check $X \leq D$ in the lattices $L\left(Z_{p^{k}}^{3}\right)$.

Now, if $p \nmid s$, then $s$ is invertible in $Z_{p^{k}}$, whence $\tilde{s}=0$. Thus, suppose $p \mid s$. If $p \mid t$ then $p \nmid u$ and $p \nmid r$ since $r, s, t$ are relatively prime and $\tilde{u}=\tilde{r}=0$ follows. If $p \nmid t$ and $p \neq 2$ then $p \nmid v$ and $\tilde{v}=0$. This leaves us to consider the case $p=2$, $s \equiv 0 \bmod 4$ and $t$ odd. Here, one has $\tilde{q}=0$ and it suffices to show $F \leq D$. But $F$ consists of the triplets $(x, y, z)$ of elements in $Z_{2^{k}}$ satisfying $z=-t y=-t x$ and $y=-(s+1) x$. Since $t$ is invertible in $Z_{2^{k}}$ it follows that $x=y$ and $(s+2) x=0$ for any such. Hence one has $F \leq F_{\phi_{s+2}}$ and it suffices to verify $F \leq D$ for lattices $L\left(Z_{2^{m}}^{3}\right)$ with $2^{m}$ dividing $s+2$. Since $4 \mid s$ by hypothesis, one gets $m=1$ and $F=Z_{2}\left(e_{1}+e_{2}+e_{3}\right) \leq Z_{2} e_{1}+Z_{2}\left(e_{2}+e_{3}\right)=D$, finally.

$$
\alpha_{12}=\alpha_{32}^{-f} \alpha_{13}^{e} \alpha_{32}^{f} \alpha_{13}^{-e} \quad \text { if } e f=1 \text {. }
$$

PROOF. This is easily checked on a suitable generating subset of $\phi$ by means of Theorem 4.1.

$$
\alpha_{i j \phi}^{e}\left(\mathbf{s}_{\phi}\right) \leq\left(\alpha_{i j}^{e} \mathbf{s}\right)_{\phi} \quad \text { for } e= \pm 1 \text { and relatively prime } s^{1}, s^{2}, s^{3} .
$$

Proof. By symmetry, (4.11), and (4.13) it suffices to consider $i=1, j=2$, $e=1, t$ odd, $s \equiv 2 \bmod 4$, and the following two cases where $\mathbf{s}=(r, s, t)$. Case I. $r$ 
is even. Case II. $r$ is odd. Choose $e=-1$ in Case I and $e$ such that $t+e r \equiv 2 \bmod 4$ in Case II. In both cases choose $f$ such that $e f=1$. Then by (4.14) and (4.13) one has

$$
\begin{aligned}
\alpha_{12}(r, s, t) & =\alpha_{32}^{-f} \alpha_{13}^{e} \alpha_{32}^{f} \alpha_{13}^{-e}(r, s, t) \leq \alpha_{32}^{-f} \alpha_{13}^{e} \alpha_{32}^{f}(r, s, t+e r) \\
& \leq \alpha_{32}^{-f} \alpha_{13}^{e}(r, s-r-f t, t+e r) \leq \alpha_{32}^{-f}(r, s-r-f t, t) \leq(r, s-r, t) .
\end{aligned}
$$

Hereby, in Case I we apply (b), (b), (a), (a) and in Case II we apply (a), Case I, (b), (c) observing $s-r-f t \equiv 0 \bmod 4$.

PROOF OF THEOREM 4.4. By (4.15) one derives $\alpha_{i j \phi}\left(\mathbf{s}_{\phi}\right) \leq\left(\alpha_{i j} \mathbf{s}\right)_{\phi}=$ $\alpha_{i j \phi} \alpha_{i j \phi}^{-1}\left(\alpha_{i j} \mathbf{s}\right) \leq \alpha_{i j \phi}(\mathbf{s})$, whence equality.

The group $\mathrm{GL}(n, Z)$ operates on $L\left({ }_{Q} Q^{n}\right)$ naturally. In particular, for each $k$ it operates transitively on the set of subspaces of dimension $k$.

LEMMA 4.5. There is an order preserving map $\kappa$ of $L\left({ }_{Q} Q^{n}\right)$ into $L$, the lattice freely generated by an $n$-frame with $\tilde{s} \leq \bar{r}(r, s>0$ integer $)$, such that $\psi_{\phi} \kappa U=\kappa \psi U$ for all $U$ in $L\left({ }_{Q} Q^{n}\right)$ and $\psi$ in $\mathrm{GL}(n, Z)$ and, moreover, $\kappa\left(\sum_{i \leq k} Q e_{i}\right)=\sum_{i \leq k} a_{i}$.

PROOF. Let $\pi U$ consist of all $\mathbf{s}$ in $Z^{n}$ belonging to $U$ with relatively prime coefficients. We claim that $\kappa U=\sum\left(\mathbf{s}_{\phi} \mid \mathbf{s} \in \pi U\right)$ exists and is mapped onto $\sum_{i \leq k} a_{i}$ under $\psi_{\phi}$ if $\psi U=\sum_{i \leq k} Q e_{i}$. Indeed, for each $\mathbf{s}$ in $\pi U$ we have, by Theorem 4.4, $\psi_{\phi} \mathbf{s}_{\phi}=\mathbf{t}_{\phi}$ with $t^{j}=0$ for $j>k$, whence $\psi_{\phi} \mathbf{s}_{\phi} \leq \sum_{i \leq k} a_{i}$ by Proposition 2.3. On the other hand $\psi^{-1} e_{i} \in \pi U$ for $i \leq k$, whence $\kappa \psi \bar{U}=\sum_{i \leq k} a_{i}=$ $\sum\left(\psi_{\phi} \mathbf{s}_{\phi} \mid \mathbf{s} \in \pi U\right)$. Therefore, $\kappa U$ exists. The compatibility with arbitrary $\psi$ follows from Theorem 4.4 immediately.

5. Frames of characteristic 0 . In this section let $L$ be generated by an $n$-frame $\phi$. If $\varphi$ is a homomorphism of $L$ onto a lattice freely generated by an $n$-frame of characteristic $m \neq 0$ then by Corollary 4.3 the restrictions of $\varphi$ to the sublattices $\left\langle\phi_{m}\right\rangle$ and $\left\langle\phi^{\bar{m}}\right\rangle$ are isomorphisms and their inverses provide the lower and upper bounds for preimages under $\varphi$. Since frames are projective configurations for modular lattices (Huhn [22]) we have that lattices generated by $n$-frames of finite characteristic $m$ are bounded homomorphic images of free lattices, splitting, and have a finite projective cover - the lattice freely generated by an $n$-frame of characteristic $m$. Of course, we refer to the variety of modular lattices-Arguesian for $n=3$.

LEMMA 5.1. For each $k$ and prime $p$ there is a subdirect decomposition of $L$ into lattices $L\left(Z_{p^{l}}^{n}\right), l<k$, and a lattice $L^{\prime}$ with image $\phi^{\prime}$ of $\phi$ such that $\left\langle\phi_{p^{k}}^{\prime}\right\rangle$ is a homomorphic image of $L\left(Z_{p^{k}}^{n}\right)$.

PROOF BY INDUCTION ON $k$. In the inductive step $k-1 \mapsto k$, let $m=p^{k-1}$. We may assume $L$ is freely generated by $\phi$ such that $\left\langle\phi_{m}\right\rangle=S$ is isomorphic to $L\left(Z_{m}^{n}\right)$. Let $M=\left\langle\phi_{p^{k}}\right\rangle$. Then by (3.5) $S$ is a sublattice of $M$ and there is an endomorphism of $L$ onto $M$ with $\varphi x \leq x$ for all $x$ in $L$. In view of Corollary 4.3 $M$ is a subdirect product of $S$ and $L\left(Z_{p^{k}}^{n}\right)$. Let $\pi_{1}$ and $\pi_{2}$ be the associated projections, $\theta$ the kernel of $\pi_{1} \varphi$. By the above, the $\theta$-classes are bounded by maps $\sigma=\mathrm{id}_{S}$ and $\gamma$. Let $\psi$ be the congruence generated by the quotients $\sigma x / \sigma x \gamma y$ where $x / y$ is a prime quotient in $S$. then $\theta \cap \psi=\operatorname{id}_{L^{--}}$cf. [32] and Proposition A.1.

To see that this yields the subdirect decomposition looked for, we only have to verify $M / \psi \mid M \cong L\left(Z_{p^{k}}^{n}\right)$. Now, $M / \theta \mid M \cong S$ and $M$ is a subdirect product of 
two factors only. Hence it suffices to show that $\psi \mid M$ is nontrivial. Let $x / y$ be a prime quotient in $S . \sigma$ and $\varphi \gamma$ are the bound maps for $\pi_{1}$ whence $\sigma x$ covers $\varphi \gamma y \sigma x$ in $M$. On the other hand $\sigma x>\gamma y \sigma x \geq \varphi \gamma y \sigma x$, whence $\gamma y \sigma x=\varphi \gamma y \sigma x$, providing a proper quotient in $\psi \mid M$.

LEMMA 5.2. The following are equivalent for given $L$ :

(i) $L$ has no subdirect factor $L\left(Z_{p^{k}}^{n}\right)$, p prime, $k<\infty$.

(ii) L satisfies all relations $\tilde{r} \leq \bar{s}, r, s>0$ integer.

(iii) $\left\langle\phi_{m}\right\rangle \subseteq L$ is a homomorphic image of $L\left(Z_{m}^{n}\right)$ for all $m>0$.

ProOF. (ii) implies (i), obviously. Assume (i) and let $m=\prod p_{i}^{k_{i}}$ the prime factor decomposition of $m$. Then $\left\langle\phi_{p_{i}^{k_{i}}}\right\rangle$ is a homomorphic image of $L\left(Z_{p_{i}^{k_{i}}}^{n}\right)$ and $\left\langle\phi_{m}\right\rangle$ of $L\left(Z_{m}^{n}\right)$ by Lemma 5.1 and 3.5. Finally, assuming (iii), let $m=r s$. Then $\tilde{r} \leq \bar{s}$ holds in $\left\langle\phi_{m}\right\rangle$ and, by $(3.5)$, in $L$ too.

LEMMA 5.3. If $L$ satisfies $\tilde{r}=0$ and $\bar{r}=1$ for all $r>0$ then $L$ is a homomorphic image of $L\left({ }_{Q} Q^{n}\right)$.

ProOF. Let $L$ be subdirectly irreducible. By (2.1) all integers are invertible in $R$ and we can apply Theorem 2.9 to the subring $S=Q$ of $R_{\phi}$.

Let $T$ denote the direct sum of the groups $Z_{p^{\infty}}$ considered as a subgroup of the unit circle and $L_{c}\left(T^{n}\right)$ the lattice of closed subgroups of $T^{n}$. Recall that $L_{c}\left(T^{n}\right)$ is dually isomorphic to $L\left(Z^{n}\right)$. In particular, it is generated by its canonical frame $\phi^{\prime}$.

LEMMA 5.4. Let $\phi$ satisfy $\tilde{r} \leq \bar{s}$ for all $r, s>0$ and let $L$ be embedded into its ideal lattice $I(L)$. Let $u_{2}$ be the ideal generated by all elements $a_{2} \tilde{r}(r>0)$, and $\phi_{u}$ the associated reduced frame. Then $u=\sum \tilde{r}$ and the sublattice $\left\langle\phi_{u}\right\rangle$ of $I(L)$ generated by $\phi_{u}$ is a homomorphic image of $L_{c}\left(T^{n}\right)$. Moreover, the $u_{p}=\sum_{k} \tilde{p}^{k}$ are independent central elements in the sublattice they generate together with $\phi_{u}$ and $u=\sum_{p} u_{p}$.

ProOF. The first claim is obvious. Now the subgroup $\tilde{m}$ of $T^{n}$ consists of all elements of order dividing $m$ and is isomorphic to $Z_{m}^{n}$. By Lemma 5.2 we have a homomorphism $\sigma_{m}$ of the interval $[0, \tilde{m}]$ of $L_{c}\left(T^{n}\right)$ into $L$ mapping the generating frame $\phi_{m}^{\prime}$ onto $\phi_{m}$. In view of (3.1) and (3.5) $\sigma_{m}$ coincides on $\phi_{r}^{\prime}$ with $\sigma_{r}$ for $r$ dividing $m$. Hence, $\sigma=\bigcup_{m} \sigma_{m}$ is a homomorphism of the lattice of finite subgroups of $T^{n}$ into $L$. Finally, since $I(L)$ is upper continuous, $\varphi S=\sum(\sigma U \mid U \subseteq S$ finite) defines a homomorphism of $L_{c}\left(T^{n}\right)$ into $I(L)$ mapping $\phi^{\prime}$ onto $\phi_{u}$ and the properties of the $u_{p}$ carry over.

REMARK 5.5. If one dualises 5.4 then $\left\langle\phi_{u}\right\rangle$ turns into the sublattice of the filter lattice $F(L)$ generated by the upper reduced frame $\phi^{v}$ arising from the filter $v_{2}$ generated by the $a_{2} \bar{r}(r>0)$. This is so since, in $L, a_{2}^{*}+\bar{r} a_{2}=a_{2}^{*}+r_{21}^{*}$ by Lemma 2.6 .

LEMMA 5.6. Let $L$ satisfy $\tilde{r} \leq \bar{s}$ for all $r, s>0$. Then $u=\sum_{r} \tilde{r} \leq v=\prod_{r} \bar{r}$ are neutral elements in $F I(L)$.

PROOF. We may assume $L$ is generated freely. Then by Lemma 5.4 and Remark $5.5\left\langle\phi_{u}\right\rangle \cong L_{c}\left(T^{n}\right)$ and $\left\langle\phi^{v}\right\rangle \cong L\left(Z^{n}\right)$. For $r>0$ we have, by $(3.1), u a_{1}+u r_{12}=$ $u\left(a_{1}+a_{2}\right)$ since $T$ is divisible. Similarily, $\left(v+a_{1}\right)\left(v+r_{12}\right)=v$ since $Z$ has no divisors 
of zero. By Jónsson [25] it follows that $u, v, a_{1}$, and $r_{12}$ generate a distributive sublattice. Therefore, $\phi_{v}^{u}=\phi^{\prime}$ satisfies $a_{1}^{\prime} r_{12 \phi^{\prime}}=u$ and $a_{1}^{\prime}+r_{12 \phi^{\prime}}=a_{1}^{\prime}+a_{2}^{\prime}$ and generates a sublattice isomorphic to $L\left({ }_{Q} Q^{n}\right)$ by Lemma 5.3. To simplify notation let us identify each of these sublattices of $L$ with the isomorphic concrete lattices such that the generating frames turn into the canonical ones.

Let $\theta$ be the congruence on $L_{c}\left(T^{n}\right)$ given by $x \theta y$ if and only if $[x y, x+y]$ has finite length. $L_{c}\left(T^{n}\right) / \theta$ is isomorphic to $L\left({ }_{Q} Q^{n}\right)$ by Lemma 5.3. Since $L_{c}\left(T^{n}\right)$ has no infinite descending chains we get a join homomorphism $\sigma$ of $L\left({ }_{Q} Q^{n}\right)$ into $L_{c}\left(T^{n}\right)$ such that $\sigma x$ is the smallest preimage of $x$ under the canonical epimorphism and $\sigma \phi^{\prime}=\phi_{u}$. Similarily, we have a canonical meet preserving map $\gamma$ of $L\left({ }_{Q} Q^{n}\right)$ into $L\left(Z^{n}\right)$ mapping $\phi^{\prime}$ onto $\phi^{v}$.

We now apply Proposition A.4 with the map $\kappa$ defined in Lemma 4.5 to the lattice generated by $L, u$, and $v$-which clearly suffices. Actually, we show that $u+v \kappa x=x, u \kappa x=\sigma x$, and $v+\kappa x=\gamma x$ for all $x$ in $L\left({ }_{Q} Q^{n}\right)$.

First, we observe that for $\psi$ in $\operatorname{GL}(n, Z)$ the automorphism $\psi_{\phi}$ of $L$ (properly, its extension to $F I(L))$ has fixpoints $u$ and $v$. Also, $\psi_{\phi}$ induces on $\left\langle\phi^{\prime}\right\rangle,\left\langle\phi_{u}\right\rangle$, and $\left\langle\phi^{v}\right\rangle$ the automorphisms which come from the action of $\psi$ on the coordinatizing modules-which has to be checked for elementary $\psi$ only. Finally, $\psi_{\phi} \sigma x=\sigma \psi x$ and $\psi_{\phi} \gamma x=\gamma \psi x$ by construction of $\sigma$ and $\gamma$.

Now, for given $x$ choose $y=\sum_{i \leq k} a_{i}^{\prime}$ and $\psi$ such that $\psi y=x$. Then $\kappa y=$ $\sum_{i \leq k} a_{i}$. By Lemmas 4.5 and 3.2 it follows that

$$
\begin{aligned}
& u+v \kappa x=\psi_{\phi}(u+v \kappa y)=\psi_{\phi} y=x, \\
& u \kappa x=\psi_{\phi}(u \kappa y)=\psi_{\phi}\left(\sum_{i \leq k} u a_{i}\right)=\sigma x
\end{aligned}
$$

and

$$
v+\kappa x=\psi_{\phi}(v+\kappa y)=\psi_{\phi}\left(\sum_{i \leq k} v+a_{i}\right)=\gamma x .
$$

PROOF OF THEOREM 1.1. Let $L$ be subdirectly irreducible and generated by an $n$-frame $\phi$. If $\phi$ has finite characteristic then Corollary 4.3 applies. Otherwise, it has characteristic 0 , and by Lemmas 5.2 and 5.6 we have neutral elements $u$ and $v$ in $F I(L)$. Since $L$ is subdirectly irreducible there are only three possible cases: $u=0, v=1$ or $u=1$ or $v=0$. In the first, Lemma 5.3 applies. Now let $u=1$. Again, by Lemma 5.4 we have $u_{p}=1$ for one $p$ and $u_{q}=0$ for all $q \neq p$, and the homomorphism $\sigma$ maps the sublattice $L_{c}\left(Z_{p^{\infty}}^{n}\right)$ of $L_{c}\left(T^{n}\right)$ onto $L$ isomorphically. Similarly, if $v=0$ we get the dual of such using Remark 5.5.

6. Frame generated subdirect products. By Theorem 1.1 every lattice $L$ generated by an $n$-frame $\phi$ of finite characteristic $m$ is a subdirect product of lattices $L\left(Z_{p^{k}}^{n}\right), p$ prime and $p^{k}$ a divisor of $m$, and the image of $\phi$ is canonical. If $m=\prod p_{i}^{k_{i}}$ then the $p_{i}^{k_{i}}$ are independent central elements and provide a direct decomposition of $L$ into factors of prime power characteristic. We have to describe the latter. For $h \leq l$ there are two embeddings, $\sigma_{p h l}$ and $\gamma_{p h l}$, of $L\left(Z_{p^{h}}^{n}\right)$ into $L\left(Z_{p^{l}}^{n}\right)$, the first coming from the canonical isomorphism of $Z_{p^{h}}^{n}$ onto the subgroup $p^{l-h} Z_{p^{l}}^{n}$, and the second from the canonical homomorphism of $Z_{p^{l}}^{n}$ onto $Z_{p^{h}}^{n}$. 
LEMMA 6.1. Let $I \subseteq N$ be finite and let $L \subseteq \times_{i \in I} L\left(Z_{p^{i}}^{n}\right)$ be a subdirect product generated by an $n$-frame $\phi$. Then $L$ consists of all sequences $\left(x_{i} \mid i \in I\right)$ with $\sigma_{p h l} x_{h} \leq x_{l} \leq \gamma_{p h l} x_{h}$ for all $h \leq l$ in $I$.

ProOF. By induction on the cardinality of $I$. Let $j$ be minimal in $I$ and $K=$ $I-\{j\}$. Let $L^{\prime}$ be the frame generated subdirect product of the $L\left(Z_{p^{k}}^{n}\right), k \in K$. Then $L$ is a subdirect product of $L\left(Z_{p^{j}}^{n}\right)$ and $L^{\prime}$ with projections $\pi$ and $\psi$. Let $\sigma$ and $\gamma$ be the two embeddings of $L\left(Z_{p^{j}}^{n}\right)$ into $L$ given by the reduced frames $\phi_{p^{j}}$ and $\phi^{\bar{p}^{j}}$. It suffices to show $(x, y) \in L$ for $\psi \sigma x \leq y \leq \psi \gamma x$. Choose $w$ in $L$ with $\psi w=y$ and let $v=(w+\sigma x) \gamma x$ in $L$. Then $\psi v=y$ and $\pi v=x$ since $\pi \sigma x=x=\pi \gamma x$.

Call an infinite matrix $\left(x_{p k}\right)$ with coefficients $x_{p k} \in L\left(Z_{p^{k}}^{n}\right)$ admissible if $\sigma_{p k h} x_{p k}$ $\leq x_{p h} \leq \gamma_{p k h} x_{p k}$ for all $k \leq h$. Call an admissible matrix low (high) if for each $p$ there is a $k$ with $\sigma_{p k h} x_{p k}=x_{p h}\left(\gamma_{p k h} x_{p k}=x_{p h}\right)$ for all $h \geq k$ and if there are only finitely many $p$ for which there is a $k$ with $x_{p k} \neq 0\left(x_{p k} \neq 1\right)$. Finally, call $\left(x_{p k}\right)$ geometric if there is a system $\Gamma$ of (in $Z^{n}$ ) independent homogeneous linear equations with relatively prime integer coefficients such that, for all $p$ and $k, x_{p k}$ is the solution set of $\Gamma$ in $Z_{p^{k}}^{n}$.

THEOREM 6.2. The lattice freely generated by an $n$-frame $\phi$ is a subdirect product of the lattices $L\left(Z_{p^{k}}^{n}\right)$ consisting of all matrices of the form $X+Y U$ with $X \leq U$, where $X$ is low, $U$ high, and $Y$ geometric. Here, $Y$ is uniquely determined.

PROOF. By Theorem 1.1 L belongs to the lattice variety generated by all subgroup lattices of abelian groups, whence it is a subdirect product of $L\left(Z_{p^{k}}^{n}\right)$ 's by $[20,4.1]$. To see that every low matrix $X$ belongs to $L$ choose $m$ such that $p^{k}$ divides $m$ if there is $h>k$ with $\sigma_{p k h} x_{p k} \neq x_{p h}$. Then by Lemma $6.1 X$ belongs to the sublattice of $L$ generated by $\phi_{m}$. Dually, we get high matrices. Observe that $Y$ is of the form "low+geometric" if and only if there is an $S$ in $L_{c}\left(T^{n}\right)$ such that $y_{p k}=S \cap Z_{p^{k}}^{n}$, considering $Z_{p^{k}}^{n}$ as a subgroup of $T^{n}$. Here, $Y$ is geometric if and only if $S$ is complemented. Hence, every geometric matrix belongs to $L$ and one sees that the meet of matrices of the form "log+geometric" is again of this form. With the dual observation it becomes clear that the matrices $X+Y U$ form a sublattice which is just $L$. If $X+Y U=X^{\prime}+Y^{\prime} U^{\prime}$ then $X+Y$ and $X^{\prime}+Y^{\prime}$ have the same image in $L_{c}\left(T^{n}\right)$, whence $Y=Y^{\prime}$.

COROLLARY 6.3. If $L$ is freely generated by an $n$-frame $\phi$ then its coordinate ring $R_{\phi}$ is the ring of integers.

Let $\sigma_{p k \infty}$ and $\gamma_{p k \infty}$ denote the canonical embeddings of $L\left(Z_{p^{k}}^{n}\right)$ into $L_{c}\left(Z_{p^{\infty}}^{n}\right)$ and $L\left(Q_{p} Q_{p}^{n}\right)$, and $\sigma_{p \infty 0}$ and $\gamma_{p \infty 0}$ the canonical homomorphism of these onto $L\left({ }_{Q} Q^{n}\right)$. Recall that for fixed $p$ every frame generated subdirect product of infinitely many $L\left(Z_{p^{k}}^{n}\right)$ 's has $L_{c}\left(Z_{p^{\infty}}^{n}\right)$ and $L\left(Q_{p} Q_{p}^{n}\right)$ as homomorphic images [20]. Hence, these lattices and $L\left({ }_{Q} Q^{n}\right)$ may occur as redundant subdirect factors. Now define the three types of matrices as above with components ranging through certain of the lattices in Theorem 1.1 such that a low matrix is 0 in $L\left({ }_{Q} Q^{n}\right)$ and all $L\left({ }_{Q_{p}} Q_{p}^{n}\right)$. Then the description given in Theorem 6.2 generalizes to arbitrary frame generated subdirect products. Moreover, let $X$ consist of those $p, p$ prime or 1 , for which $L\left(Q_{p} Q_{p}^{n}\right)$ or its dual is a subdirect factor of $L$. Let $Y$ consist of all $p^{k}>1, p \notin X$ 
and $k$ maximal, such that $L\left(Z_{p^{k}}^{n}\right)$ is a subdirect factor of $L$. Then, denoting by $Q_{X}$ the ring of all rationals with denominator prime to all $p$ in $X$, we get

COROLLARY 6.4: The coordinate ring $R_{\phi}$ of $L$ generated by $\phi$ is $Z_{m} \times Q_{X}$ if $Y$ is finite, $m=\prod Y$ and, else, $Q_{X \cup Y}$.

THEOREM 6.5. Let $L$ be a lattice of finite length with $n$-frame $\phi$. Then $L$ is isomorphic to the direct product of finitely many lattices $L_{i}$ with $n$-frames $\phi_{i}$ each of which has prime power characteristic or $Q$ as a subring of $R_{\phi_{i}}$.

The explicit formulation of this result is due to Ralph Freese, who also gave a proof independent of Theorem 1.1 and observed that, by the dimension formula for $r$ in $R_{\phi}$,

$$
a_{1} r_{12}=0 \text { if and only if } a_{1}+r_{12}=a_{1}+a_{2} .
$$

PROOF. By Theorem 1.1 the sublattice $L^{\prime}$ generated by $\phi$ is a subdirect product of finitely many lattices $L\left(Z_{p^{k}}^{n}\right), p$ prime, $k<\infty$, and maybe $L\left({ }_{Q} Q^{n}\right)$. For a fixed prime $p$ choose $k$ maximal such that $L\left(Z_{p^{k}}^{n}\right)$ is a subdirect factor of $L^{\prime}$. Then, $\phi_{p^{k}}$ has characteristic $p^{k}$ and $p$ is invertible in the coordinate ring $R_{\phi_{\bar{p}^{k}}}$ in $L$-since it is so in $L^{\prime}$. Also, $\tilde{p}^{k}$ and $\bar{p}^{k}$ are complements. (For that Freese gives a direct proof choosing $k$ maximal with $\tilde{p}^{k} \neq \tilde{p}^{k+1}$.) Of course, it suffices to show (for each $p$ ) that $\tilde{p}^{k}$ and $\bar{p}^{k}$ are central elements in $L$.

Assume the contrary. Then by Proposition A.5 there are $i$ and $j$ such that $\tilde{p}^{k} a_{i}$ and $\bar{p}^{k} a_{j}$ are not central in $\left[0, \tilde{p}^{k} a_{i}+\bar{p}^{k} a_{j}\right]$, and in view of the given perspectivities we may assume $i=j=1$. Let $x$ witness this fact, i.e. with $u_{1}=x \tilde{p}^{k} a_{1}+x \bar{p}^{k} a_{1}$, $v_{1}=\left(x+\tilde{p}^{k} a_{1}\right)\left(x+\bar{p}^{k} a_{1}\right), y_{1}=\left(u_{1}+\tilde{p}^{k} a_{1}\right) v_{1}$, and $z_{1}=\left(u_{1}+\bar{p}^{k} a_{1}\right) v_{1}$, we get that $u_{1}<x, y_{1}, z_{1}<v_{1}$ form a sublattice $M_{3}$ of $L$. Since $L$ has finite length we may assume $v_{1}$ covers $x$, and $x$ covers $u_{1}$ in $L$.

Now consider the reduced frames $\phi_{v}^{u}, \phi_{y}^{u}$, and $\phi_{z}^{u} \cdot y=u+v \tilde{p}^{k}$ and $\phi_{y}^{u}$ arises from $\phi_{p^{k}}$, whence it has characteristic dividing $p^{k}$ by Corollary 3.4. Similarly, $z=u+v \tilde{p}^{k}$ and $\phi_{z}^{u}$ arises from $\phi_{\bar{p}^{k}}$, which implies by (6.1) and (2.1) that $p$ is invertible in $R_{\phi_{z}^{u}}$. Also, $y$ and $z$ are complements in $[u, v]$. On the other hand $y_{1}+u$ and $z_{1}+u$ cover $u$ and are perspective via $x+u$. Hence $\phi_{y}^{u}$ and $\phi_{z}^{u}$ provide $2 n$ independent atoms of $[u, v]$, all of which are perspective. This means $[u, v]$ is an irreducible $(2 n-1)$ dimensional projective geometry and can be coordinatized over a skew field $F$. In particular, the intervals $[u, y]$ and $[u, z]$ can both be coordinatized over $F$, and $\phi_{y}^{u}$ and $\phi_{z}^{u}$ must have the same characteristic - that of $F$. This is a contradiction since $p$ is 0 in $R_{\phi_{y}^{u}}$ and invertible in $R_{\phi_{z}^{u}}$.

7. Finite basis versus finite generation. The proof of the Theorem 1.4 consists of constructing a family of lattices $L_{p q}, p$ and $q$ distinct primes, none of which belongs to the variety generated by finite length modular lattices, but an ultrapower of which belongs to the lattice variety $M_{0}$ generated by rational projective geometries.

To define $L_{p q}$ let $p$ and $q$ be fixed and $n \geq 4$. Let $R_{p^{*}}$ denote the ring of $p$-adic integers, $V_{p}$ the rank $n$ free module over this ring, and $L_{1}$ its lattice of submodules. Factorizing $L_{1}$ by the smallest lattice congruence which identifies all elements of finite dual rank (i.e. all submodules of finite index) we obtain an $(n-1)$ dimensional projective geometry over a field $F$ of characteristic 0 - the reasoning in 
$[20,4.5]$ carries over. Since $L_{1}$ satisfies the ascending chain condition each $\theta$-class contains an upper bound. This establishes a meet preserving map $\gamma$ of $L\left(F_{F}^{n}\right)$ into $L_{1}$ matching the canonical frames. Also, observe that the $\theta$-class of $\gamma x$ consists of all submodules $U$ of $V_{p}$ for which the quotient $\gamma x / U$ is finite. Therefore, each $\theta$-class is countable and, $L_{1}$ being uncountable, $F$ must also be uncountable.

Actually, it is not hard to see that $F$ is the field of $p$-adic numbers and that the homomorphism of $L_{1}$ onto $L_{1} / \theta$ is given by $U \mapsto U \otimes_{R_{p^{*}}} F$. Just note that this homomorphism induces a ring homomorphism of $R_{p^{*}}=R_{12 \phi}-\phi$ the canonical frame of $L_{1}$-with kernel $a_{1}$ and that $F$ is flat as a $R_{p^{*}}$-module.

Now let $S$ be the nonmodular lattice $L\left(\mathbf{Q}_{\mathbf{Q}}^{n}\right)$ with an extra element $e$ added which is between 0 and 1 and a complement of any other. There is a meet embedding of $S$ into $L\left(F_{F}^{n}\right)$ which is the canonical embedding on the rational projective geometry and sends $e$ to a point which is on no rational hyperplane. For example, $e$ can be sent to a one-dimensional subspace spanned by a vector in $F^{n}$ whose coordinates are linearly independent over $\mathbf{Q}$. Using the above, one obtains a meet embedding, also denoted by $\gamma$, of $S$ into $L_{1}$ which matches the canonical frames and maps $e$ onto a rank 1 free submodule of $V_{p}$. Thus, the interval $[0, \gamma e]$ is dually isomorphic to the ordinal $\omega+1$.

Let $L_{0}$ be the dual of $L\left(V_{q}\right)$ and the join preserving map $\sigma$ of the dual $S^{\delta}$ of $S$ into $L^{0}$ be defined dually. Identify $S$ with $S^{\delta}$ in such a way that $\sigma$ matches the canonical frames of $L\left(\mathbf{Q}_{\mathbf{Q}}^{n}\right) \subseteq S$ and $L_{0}$. By construction, $[\sigma e, 1]$ in $L_{0}$ is isomorphic to $\omega+1$. Now consider the subset

$$
A_{p q}=\bigcup_{x \in S}[(\sigma x, 0),(1, \gamma x)]
$$

of $L_{0} \times L_{1}$ which is obviously a sublattice. Observe that $[(\sigma e, 0),(1, \gamma e)] \cong \omega+1 \times$ $(\omega+1)^{\delta}$ with complementary central elements $(1,0)$ and $(\sigma e, \gamma e)$. Let $\bar{e}$ and $\underline{e}$ be the upper and lower covers of $\sigma e$ and $\gamma e$ in $L_{0}$ and $L_{1}$, respectively. Obtain $L_{p q}$ from $A_{p q}$ by fitting in a new element $e_{0}$ into the interval $[(\sigma e, \underline{e}),(\bar{e}, \gamma e)]=\left[e_{1}, e_{2}\right]$, which is a complement of both $e_{3}=(\sigma e, \gamma e)$ and $e_{4}=(\bar{e}, \underline{e})$, therein. This yields a modular lattice $L_{p q}$. Denote by $p^{+}$the prime succeeding $p$.

LEMMA 7.1. Every nontrivial ultraproduct of $L_{p q^{+}}$'s belongs to the lattice variety generated by rational projective geometries.

Let us take care of the $A_{p p^{+}}$'s first. There is an axiomatic correspondence between these lattices and the modules $V_{p}, V_{p^{+}}$(together with their rings $R_{p^{*}}$ and $R_{p^{+*}}$ ) expressing that $A_{p p^{+}}$is embedded into the direct product of $L\left(V_{p}\right)$ and the dual of $L\left(V_{p^{+}}\right)$.

Now let $A$ be an ultraproduct of the $A_{p p^{+}}$'s over a nonprincipal ultrafilter, $V$ and $R$ the ultraproducts of the $V_{p}$ and $R_{p^{*}}$ over the same filter-which happen to be the ultraproducts of the $V_{p^{+}}$and $R_{p^{+*}}$ too. Then, in view of the axiomatic correspondence, $A$ is embedded into the direct product of $L(V)$ and its dual, $L(V)$ being the lattice of $R$-submodules of $V$. But in $R$ every prime is invertible, which means $\mathbf{Q}$ is a subring of $R$ and we may consider $V$ as a $\mathbf{Q}$-module too, and $L\left(V_{R}\right)$ as a sublatice of $L\left(V_{\mathbf{Q}}\right)$. Thinking of the dual of $L\left(V_{\mathbf{Q}}\right)$ as being embedded into the subspace lattice $L\left(V_{\mathbf{Q}}^{*}\right)$ of the vector space $V_{\mathbf{Q}}^{*}$ dual to $V_{\mathbf{Q}}$, we have an embedding of $A$ into a direct product $L\left(W_{1}\right) \times L\left(W_{2}\right), W_{1}$ and $W_{2}$ vector spaces over $\mathbf{Q}$. 
Let $L$ be the corresponding ultraproduct of the $L_{p p^{+}}$. Then $A$ is a sublattice of $L$. Add constants $\sigma e, \gamma e, \bar{e}, \underline{e}, e_{i}(0 \leq i \leq 4)$ to the language denoting the abovementioned particular elements in each lattice $L_{p q}$. Then the covering relations between these are valid in $L$ too, and the $e_{i}$ form a sublattice $M_{3}$. Also, $A$ is just $L$ with $e_{0}$ removed. Clearly, we can choose the vector spaces $W_{1}$ and $W_{2}$ such that the quotients $\bar{e} / \sigma e$ and $\gamma e / \underline{e}$ have the same dimension. Having $L\left(W_{1}\right) \times L\left(W_{2}\right)$ canonically embedded into $L\left(W_{1} \oplus W_{2}\right)$, we have $A$ embedded therein too, and may now choose for $e_{0}$ any of the common complements of $e_{3}$ and $e_{4}$ in the interval $\left[e_{1}, e_{2}\right]$ of $L\left(W_{1} \oplus W_{2}\right)$ to end up with an embedding of $L$ into the subspace lattice of a rational projective geometry.

LEMMA 7.2. No lattice $L_{p q}$ belongs to a lattice variety generated by modular lattices of finite length.

ProOF. Assume the contrary, i.e. there is a subdirect product $M$ of modular lattices of finite length having $L_{p q}$ as a homomorphic image. Now, $L_{p q}$ has a spanning frame which is canonical in both components of $A_{p q}$ and $a_{1} \tilde{q} / 0$ is projective to $1 / a_{1}^{*}+\bar{p}$ via a projectivity passing through the interval $\left[e_{1}, e_{2}\right]$. According to Huhn [22] one can find a frame $\phi: a_{i}, c_{i j}$ in $M$ which is mapped onto this canonical frame. By Wille $[\mathbf{3 3}]$ there is a proper quotient in $M$ too, weakly projective into $a_{1} \tilde{q} / 0$ and $1 / a_{1}^{*}+\bar{p}$ now taken in $M$ with respect to $\phi$. Clearly, we may assume $\sum \phi=1$ and $\prod \phi=0$. All this carries over to a subdirect factor $L$ of $M$ in which this proper quotient is separated.

In other words, we have a subdirectly irreducible modular lattice $L$ of finite length with spanning frame $\phi: a_{i}, c_{i j}$ and a proper quotient $x / y$ weakly projective into both $a_{1} \tilde{q} / 0$ and $1 / a_{1}^{*}+\bar{p}$. By Theorem 6.5 either $q$ or $p$ is invertible in the coordinate ring. In both cases one of the quotients collapses in view of $(2.1)$, whence $x / y$ collapses too, a contradiction.

Now if $V$ is a finitely based variety containing $M_{0}$ we have $L_{p q}$ in $V$ for suitable $p$ and $q$, by Lemma 7.1, and $V$ cannot be generated by its finite-dimensional members in view of Lemma 7.2. Thus we have proven Theorem 1.4.

\section{Appendix. Subdirect decomposition methods.}

Proposition A.1. Let $L$ and $M$ be modular lattices, $L$ of finite length and $M$ subdirectly irreducible. Let $\gamma(\sigma)$ be a meet (join) homomorphism of $L$ into $M$. Suppose $\sigma x \leq \gamma x$ for all $x$ in a generating set of $L$, and $M$ is generated by the union of all intervals $[\sigma x, \gamma x], x$ in $L$. Then either $\sigma=\gamma$ is a homomorphism of $L$ onto $M$ (and an isomorphism if $L$ is subdirectly irreducible) or $\sigma p \leq \gamma q$ holds for all prime quotients $p / q$ of $L$.

This is Proposition 7 in Wille [32] for a subdirectly irreducible $L$ and Proposition 1 in [16]. Just observe that $\sigma x \leq \gamma x$. Commonly, one drops the subdirect irreducibility of $M$ and thinks of $M$ being freely generated under certain relations, $L$ a particular model and $\sigma x \leq \gamma x$ the bounds of the preimage of $x$ under the canonical epimorphism. Then, a prime quotient of $L$ is associated with the prime quotient $\sigma p+\gamma q / \gamma q$ of $M$, the congruence identifying this quotient and its pseudocomplement. This method also appears in McKenzie [30] and Freese [9]. Also we use subdirect decompositions via neutral elements. The basic method has been stated in [16, Proposition 2]. 
Proposition A.2. Let $M$ be a modular lattice, $u$ an element of $M, S$ a maybe nonmodular lattice, and $\alpha$ an order preserving map of $S$ into $M$ such that $M$ is generated by $u$ and the image of $\alpha, x \mapsto u \alpha x$ preserves joins, and $x \mapsto u+\alpha x$ preserves meets. Then $u$ is neutral in $M$.

Proposition A.3. Let $u \leq v$ be elements of a modular lattice $M$. Let $S$ be a lattice, $\sigma$ a join homomorphism and $\gamma$ a meet homomorphism of $S$ into $M$ such that $u+\sigma x=v \gamma x$ for all $x$ in a generating subset of $S$. Assume $x \mapsto u+\sigma x$ and $x \mapsto v \gamma x$ are homomorphisms of $S$ into $M$, and $M$ is generated by the union of all intervals $[\sigma x, \gamma x], x$ in $S$. Then $M$ decomposes subdirectly into a factor with $u=v$ and another one with $\sigma x=\gamma x$ for all $x$ in $S$.

ProOF. Clearly, $u+\sigma x=v \gamma x$ for all $x$ in $S$ since this holds on a generating set. Let $\varphi$ denote this homomorphism and $S^{\prime}$ the factor lattice $S / \operatorname{ker} \varphi$. Consider $M$ being embeddded into its filter lattice $F(M)$ and this embedded into its ideal lattice $I F(M)$. Observe that for a downward directed set $X \subseteq M$ and for $a$ in $F(M)$ we have $a+\prod X=\prod(a+x \mid x \in X)$ since $F(M)$ is a lower continuous lattice and the embedding of $F(M)$ into $I F(M)$ preserves arbitrary meets. For an upward directed set $X \subseteq M$ and an $a$ in $I F(M)$, we have $a \sum X=\sum(a x \mid x \in X)$ since $I F(M)$ is an upper continuous lattice.

Now define, for $X$ in $S^{\prime}, \sigma^{\prime} X=\prod(\sigma x \mid x \in X)$ and $\gamma^{\prime} X=\sum(\gamma x \mid x \in X)$. Then $\sigma^{\prime}$ is a join and $\gamma^{\prime}$ a meet homomorphism of $S^{\prime}$ into $I F(M)$ and one has $u+\sigma^{\prime} X=u+\sigma x=v \gamma y=v \gamma^{\prime} X$ for all $X$ in $S^{\prime}$ and $x, y$ in $X$. Thus, $\psi X=u+\sigma^{\prime} X$ defines an injective homomorphism of $S^{\prime}$ into $I F(M)$ since $\psi X=\psi Y$ implies $\varphi x=\varphi y$ for $x$ in $X$ and $y$ in $Y$, i.e. $X=Y$.

Let $M^{\prime}$ be the sublattice of $I F(M)$ generated by the union of $M$, the image of $\sigma^{\prime}$, and the image of $\gamma^{\prime}$. Define a relation $\theta$ on $M^{\prime}$ by

$$
a \theta b \Leftrightarrow \exists \text { an } X \text { in } S^{\prime} \text { with } \sigma^{\prime} X \leq a, b \leq \gamma^{\prime} X .
$$

We claim $\theta$ is a congruence relation on $M^{\prime}$ with classes $\left[\sigma^{\prime} X, \gamma^{\prime} X\right], X$ in $S^{\prime}$. Namely, assume $a \in\left[\sigma^{\prime} X, \gamma^{\prime} X\right] \cap\left[\sigma^{\prime} Y, \gamma^{\prime} Y\right]$. Put $Z=X+Y$. Then $\sigma^{\prime} Z \leq a \leq \gamma^{\prime} X$, whence $\psi Z=u+\sigma^{\prime} Z=v \gamma^{\prime} X=\psi X$. On the other hand $\psi X \leq \psi Z$ since $X \leq Z$. It follows that $\psi X=\psi Z$ and $X=Z$ by the injectivity of $\psi$. Symmetrically, we get $Y=Z$, whence $X=Y$. Thus, $\theta$ is a congruence relation on $M$. Then, the restriction $\theta \mid M$ is a congruence on $M$. By construction, $a \theta b$ and $u \leq a \leq b \leq v$ jointly imply $u+\sigma x \leq a \leq b \leq v \gamma x$ for suitable $x$ in $S$, whence $a=b$.

Let $\tau$ denote the congruence on $M$ generated by $v / u$. Then $\tau \cap \theta \mid M$ is the identical congruence $\operatorname{id}_{M}$ on $M$. Otherwise there would be $d>c$ with $c \tau d$ and $c \theta d$; but $c \tau d$ implies there is a proper subquotient $b / a$ of $v / u$ projective to a subquotient of $d / c$ (see [4, 10.2 and 10.3], whence $a \theta b$, too, a contradiction. This yields the subdirect decomposition we have looked for.

Proposition A.4. Let $M$ be a modular lattice and $u \leq v$ elements of $M$. Then $u$ and $v$ are neutral in $M$ provided there are a lattice $S$ and an order preserving map $\alpha$ of $S$ into $M$ having the following properties: $\alpha 0=0 ; \alpha 1=1$; the maps $x \mapsto u \alpha x, x \mapsto v+\alpha x, x \mapsto u+v \alpha x=v(u+\alpha x)$ are a join homomorphism, meet homomorphism, and homomorphism, respectively; $M$ is generated by $u, v$ and the image of $\alpha$. 
Proof. Applying Proposition A.2 to $S$ and the map $x \mapsto v \alpha x$ we have that $u$ is neutral in the sublattice it generates together with all $v \alpha x(x$ in $S)$. Similarily, $v$ is neutral in the sublattice generated by $v$ and the $u+\alpha x(x$ in $S)$. Now let $S^{\prime}$ be the modular lattice freely generated by the set $\{u, v\} \cup S$ and $\sigma, \gamma$ the homomorphisms of $S^{\prime}$ into $M$ with $\sigma u=\gamma u=u, \sigma v=\gamma v=v, \sigma x=v \alpha x$, and $\gamma x=u+\alpha x$ for $x$ in $S$. By Proposition A.3 we have a subdirect decomposition of $M$ into a factor with $u=v$ and a factor with $\sigma x=\gamma x$ for all $x$ in $S^{\prime}$. For the first, Proposition A.2 yields the neutrality of $u=v$ immediately. In the second we have $u=0$ and $v=1$ substituting $x=0_{S}$ and $x=1_{S}$ in $\sigma x=\gamma x$. Hence, $u$ and $v$ are neutral in $M$ too.

Proposition A.5. Let $a_{1}, \ldots, a_{n}, b_{1}, \ldots, b_{m}$ be an independent set of elements in a modular lattice $M$ such that for all $i$ and $j$ the elements $a_{i}$ and $b_{j}$ are central in the interval $\left[0, a_{i}+b_{j}\right]$ of $M$. Then $\sum a_{i}$ and $\sum b_{j}$ are complementary central elements in the interval $\left[0, \sum a_{i}+\sum b_{j}\right]$.

The proof is by induction on $n+m$. Considering the case $n=2, m=1$, only, write $a=a_{1}, b=a_{2}, c=b_{1}$ and let $d$ be an arbitrary element of $[0, a+b+c]$. By hypothesis we have the relations

$$
d(b+c)=b d+c d \text { and } a(b+d)+c(b+d)=(a+c)(b+d) .
$$

Consequently,

$$
\begin{aligned}
a+b+c d & =a+b+d(b+c)=a+b+(b+d)(b+c) \geq a+b+c(b+d) \\
& =a+b+(a+c)(b+d)=(a+b+d)(a+b+c) \geq d
\end{aligned}
$$

and $d(a+b)+d c=d(a+b+d c)=d$, showing that $d$ distributes with $a+b$ and $c$.

\section{REFERENCES}

1. F. W. Anderson and K. R. Fuller, Rings and categories of modules, Springer, New York, 1973.

2. B. Artmann, On coondinates in modular lattices, Illinois J. Math. 12 (1968), 626-648.

3. G. Birkhoff, Lattice theory, 3rd ed., Amer. Math. Soc. Colloq. Publ., vol. 25, Amer. Math. Soc., Providence, R.I., 1967.

4. P. Crawley and R. P. Dilworth, Algebraic theory of lattices, Prentice-Hall, Englewood Cliffs, N.J., 1973.

5. A. Day, Modular lattices and projective geometry, Lecture Notes, Lakehead Univ., 1980.

6. __ Equational theories of projective geometries, Colloq. Math. Soc. János Bolyai 33 (1983), 277-316.

7. __ A lemma on projective geometries as modular and/or Arguesian lattices, Canad. Math. Bull. 26 (1983), 283-290.

8. A. Day and D. Pickering, The coordinatization of Arguesian lattices, Trans. Amer. Math. Soc. 278 (1983), 507-522.

9. R. Freese, Breadth two modular lattices, Proc. Univ. Houston Conf. Lattice Theory, Houston, 1973.

10. __ Projective geometries as projective modular lattices, Trans. Amer. Math. Soc. 251 (1979), 329-342.

11. Soc. 255 (1979), 277-300.

12. __ Free modular lattices, Trans. Amer. Math. Soc. 261 (1980), 81-91.

13. R. Freese and B. Jónsson, Congruence modularity implies the Arguesian identity, Algebra Universalis 6 (1976), 225-228.

14. K. Fryer and I. Halperin, The von Neumann coondinatization theorem for complemented modulas lattices, Acta Sci. Math. (Szeged) 17 (1956), 203-249. 
15. L. Fuchs, Infinite abelian groups, Academic Press, New York, 1970.

16. C. Herrmann, On modular lattices generated by two complemented pairs, Houston J. Math. 2 (1976), 513-523.

17. __ Rahmen und erzeugende Quadrupel in modularen Verbänden, Algebra Universalis 14 (1982), 357-387.

18. __ On the word problem for the modular lattice with four free generators, Math. Ann. 265 (1983), 513-527.

19. C. Herrmann and A. Huhn, Zum Wortproblem für freie Untermodulverbände, Arch. Math. 26 (1975), 449-453.

20. __ Lattices of normal subgroups generated by frames, Colloq. Math. Soc. János Bolyai 14 (1975), 97-136.

21. C. Herrmann, M. Kindermann and R. Wille, On modular lattices generated by $1+2+2$, Algebra Universalis 5 (1975), 243-251.

22. A. Huhn, Schwach distributive Verbände. I, Acta Sci. Math. (Szeged) 33 (1972), 297-305.

23. _ On G. Grätzer's problem concerning automorphisms of a finitely presented lattice, Algebra Universalis 5 (1975), 65-71.

24. G. Hutchinson, Embedding and unsolvability theorems for modular lattices, Algebra Universalis 7 (1977), 47-84.

25. B. Jónsson, Distributive sublattices of a modular lattice, Proc. Amer. Math. Soc. 6 (1955), 682-688.

26. B. Jónsson and G. Monk, Representation of primary Arguesian lattices, Pacific J. Math. 30 (1969), 95-139.

27. B. Jónsson, The class of Arguesian lattices is self-dual, Algebra Universalis 2 (1972), 396.

28. F. Maeda, Kontinuierliche Geometrien, Springer, Berlin, 1958.

29. B. R. McDonald, Geometric algebra over local rings, Dekker, New York, 1976.

30. R. McKenzie, Equational bases and nonmodular lattice varieties, Trans. Amer. Math. Soc. 174 (1972), 1-43.

31. J. von Neumann, Continuous geometry, Princeton Univ. Press, Princeton, N.J., 1960.

32. R. Wille, On modular lattices generated by finite chains, Algebra Universalis 3 (1973), 131-138.

33. __ On primitive subsets of lattices, Algebra Universalis 2 (1972), 95-98.

FaChBereich Mathematik, TeChnische Hochschule DaRmstadT, 6100 DarmSTADT, SCHLOSSGARTENSTRASSE 7, WEST GERMANY 